UFIFT-QG-05-07

gr-qc/0508015

\title{
Charged Scalar Self-Mass during Inflation
}

\author{
E. O. Kahya ${ }^{\dagger}$ and R. P. Woodard ${ }^{\ddagger}$ \\ Department of Physics \\ University of Florida \\ Gainesville, FL 32611 USA
}

\begin{abstract}
We compute the one loop self-mass of a charged massless, minimally coupled scalar in a locally de Sitter background geometry. The computation is done in two different gauges: the noninvariant generalization of Feynman gauge which gives the simplest expression for the photon propagator and the de Sitter invariant gauge of Allen and Jacobson. In each case dimensional regularization is employed and fully renormalized results are obtained. By using our result in the linearized, effective field equations one can infer how the scalar responds to the dielectric medium produced by inflationary particle production. We also work out the result for a conformally coupled scalar. Although the conformally coupled case is of no great physical interest the fact that we obtain a manifestly de Sitter invariant form for its self-mass-squared establishes that our noninvariant gauge introduces no physical breaking of de Sitter invariance at one loop order.
\end{abstract}

PACS numbers: $4.62 .+\mathrm{v}, 98.80 . \mathrm{Cq}$

† e-mail: emre@phys.ufl.edu

¥ e-mail: woodard@phys.ufl.edu 


\section{Introduction}

Quantum loop effects can be understood in very simple terms as the response of classical field theory to the 0-point fluctuations of the various dynamical variables. These fluctuations can be viewed as virtual particles which emerge from the vacuum and disappear back into it after a period whose duration is fixed by the energy-time uncertainty principle. Because spacetime expansion redshifts virtual particle energies it increases the time for which they can persist, hence strengthening the effects they produce. Parker was the first to perform serious computations about this [1].

Just as in flat space, the persistence time for virtual particles of the same momentum becomes longer as the mass decreases. It is simple to show that any massless and sufficiently long wavelength virtual particle which happens to emerge from the vacuum can persist forever during inflation [2, 3, 4. However, this will not lead to increased quantum effects unless the rate at which such particles emerge from the vacuum is significant. Almost all masslessless particles possess classical conformal invariance, which causes the rate at which they emergence from the vacuum to redshift so rapidly that there is no significant strengthening of quantum effects [2, 3, 4. The two exceptions are massless, minimally coupled (MMC) scalars and gravitons. These particles are produced copiously during inflation and can therefore mediate enhanced quantum effects.

It is quantum fluctuations of precisely these fields which are responsible for the primordial cosmological scalar [5] and tensor [6] perturbations predicted by inflation [7, 8. Those are tree order effects. At one loop order it has recently been discovered that MMC scalars can catalyze significant quantum effects involving particles which would not otherwise experience them owing to classical conformal invariance. When electromagnetism (which is conformally invariant for $D=4$ dimensions) is coupled to a charged MMC scalar the resulting one loop vacuum polarization causes super-horizon photons to behave, in some ways, as though they have nonzero mass 9, 10, 11. When massless fermions (which are conformally invariant in any dimension) are Yukawa coupled to a MMC scalar the resulting one loop fermion self-energy engenders a faster-than-exponential growth in the fermion mode functions which seems to betoken explosive particle production [12].

Both models have great phenomenological interest because all Standard Model particles would be effectively massless on the energy scales typically envisaged for primordial inflation, and because they couple to a fundamental 
scalar — the Higgs — which may well be minimally coupled. If unchecked, the Yukawa process would lead to a degenerate fermi gas of super-horizon fermions [12. The electromagnetic process would not result in photon creation during inflation but leads instead to a vast enhancement of the 0-point energy of super-horizon photons. After the end of inflation the least superhorizon of these modes will re-enter the horizon and again become massless, at which point their excess 0 -point energies may seed cosmic magnetic fields [13, 14, 2].

Both effects require the scalar to be massless on the scale of the inflationary Hubble constant and it is natural to wonder whether quantum corrections can affect this. What happens can be determined by computing the self-masssquared and using this to solve the linearized effective field equations,

$$
\partial_{\mu}\left(\sqrt{-g} g^{\mu \nu} \partial_{\nu} \varphi\right)-\int d^{4} x^{\prime} M^{2}\left(x ; x^{\prime}\right) \varphi\left(x^{\prime}\right)=0 .
$$

In a previous paper it was shown that the scalar cannot acquire a large enough mass rapidly enough to prevent the Yukawa process from going to completion [15]. That result was inevitable because only the conformally invariant fermion propagator contributes to the Yukawa self-mass-squared at one loop order. The non-conformal scalar propagator participates even at one loop order in SQED so there should be a strong effect. Because inflation induces an effective dielectric medium of super-horizon charged scalars [2], and because the energy of a charged particle is reduced in a dielectric medium, it is conceivable that the effect will be to enhance inflationary particle production. In this paper we evaluate $M^{2}\left(x ; x^{\prime}\right)$ at one loop; we will use it in a subsequent paper to solve the effective field equation (11).

Because this computation involves the photon propagator we must face the issue of gauge fixing. It turns out that the simplest gauge breaks de Sitter invariance [16]. Because the scalar propagator must show a physical — as opposed to gauge - breaking of de Sitter invariance [17] this should not be an issue. However, one might worry about the introduction of spurious violations of de Sitter invariance. That can be checked by computing the self-mass-squared of the conformally coupled scalar, which shows no physical breaking of de Sitter invariance and for which we obtain a manifestly de Sitter invariant result. Another check is by computing the minimally coupled scalar self-mass-squared in the de Sitter invariant gauge of Allen and Jacobson [18.

In the next section we use the Lagrangian of SQED to derive the Feynman rules in the simplest gauge. In section 3 we compute the fully renormalized 
one loop scalar self-mass-squared. In section 4 the same quantity is computed in the de Sitter invariant gauge of Allen and Jacobson. What it all means is discussed in section 5 .

\section{Feynman Rules}

Let $\varphi(x)$ represent a complex scalar field and let $A_{\mu}(x)$ stand for the vector potential. The Lagrangian of MMC scalar QED is,

$$
\begin{aligned}
\mathcal{L}_{\mathrm{SQED}}=-\left(\partial_{\mu}-i e_{0} A_{\mu}\right) \varphi^{*} & \left(\partial_{\nu}+i e_{0} A_{\nu}\right) \varphi g^{\mu \nu} \sqrt{-g} \\
& -\xi_{0} \varphi^{*} \varphi R \sqrt{-g}-\frac{1}{4} F_{\mu \nu} F_{\rho \sigma} g^{\mu \rho} g^{\nu \sigma} \sqrt{-g} .
\end{aligned}
$$

Here $\xi_{0}$ is the bare conformal coupling and $e_{0}$ is the bare charge. We do not need to include a bare mass because mass is multiplicatively renormalized in dimensional regularization. We would need a $\left(\varphi^{*} \varphi\right)^{2}$ interaction for strict renormalizability but we shall not require such a term for this computation.

Renormalization is organized in the usual way. We first define renormalized fields in terms of the bare ones,

$$
\varphi \equiv \sqrt{Z}_{2} \varphi_{r} \quad \text { and } \quad A_{\mu} \equiv \sqrt{Z}_{3} A_{r \mu} .
$$

In terms of the renormalized fields the Lagrangian takes the form,

$$
\begin{aligned}
\mathcal{L}_{\mathrm{SQED}}=-Z_{2}\left(\partial_{\mu}-i e_{0} \sqrt{Z}_{3} A_{r \mu}\right) \varphi_{r}^{*} i\left(\partial_{\nu}+i e_{0} \sqrt{Z_{3}} A_{r \nu}\right) \varphi_{r} g^{\mu \nu} \sqrt{-g} \\
-\xi_{0} Z_{2} \varphi_{r}^{*} \varphi_{r} R \sqrt{-g}-\frac{1}{4} Z_{3} F_{r \mu \nu} F_{r \rho \sigma} g^{\mu \rho} g^{\nu \sigma} \sqrt{-g} .
\end{aligned}
$$

We will henceforth speak only of the renormalized fields and, because there can be no more ambiguity with unrenormalized fields, we will dispense with the superfluous subscript " $r$ ". Because of gauge invariance and the fact that we want the tree order scalar to be minimally coupled our renormalized parameters are given by the relations,

$$
\sqrt{Z}_{3} e_{0}=e+0 \quad \text { and } \quad Z_{2} \xi_{0}=0+\delta \xi .
$$

Setting $Z_{2,3}=1+\delta Z_{2,3}$, we finally express the Lagrangian as,

$$
\begin{array}{r}
\mathcal{L}_{\mathrm{SQED}}=-\left(\partial_{\mu}-i e A_{\mu}\right) \varphi^{*}\left(\partial_{\nu}+i e A_{\nu}\right) \varphi g^{\mu \nu} \sqrt{-g}-\frac{1}{4} F_{\mu \nu} F_{\rho \sigma} g^{\mu \rho} g^{\nu \sigma} \sqrt{-g} \\
-\delta Z_{2}\left(\partial_{\mu}-i e A_{\mu}\right) \varphi^{*}\left(\partial_{\nu}+i e A_{\nu}\right) \varphi g^{\mu \nu} \sqrt{-g}-\delta \xi \varphi^{*} \varphi R \sqrt{-g} \\
-\frac{1}{4} \delta Z_{3} F_{\mu \nu} F_{\rho \sigma} g^{\mu \rho} g^{\nu \sigma} \sqrt{-g} \cdot
\end{array}
$$


Although we can only obtain propagators in very special geometries it is simple enough to read off the interactions needed to compute the one loop self-mass-squared in a general metric background. The 3-point and 4-point interactions are,

$$
\begin{aligned}
& \mathcal{L}_{3 \mathrm{pt}}=i e A_{\mu}\left(\varphi^{*} \varphi_{, \nu}-\varphi_{, \nu}^{*} \varphi\right) g^{\mu \nu} \sqrt{-g} \\
& \mathcal{L}_{4 \mathrm{pt}}=-e^{2} A_{\mu} A_{\nu} \varphi^{*} \varphi g^{\mu \nu} \sqrt{-g}
\end{aligned}
$$

The counterterms we shall need are,

$$
\mathcal{L}_{\mathrm{ctm}}=-\delta Z_{2} \varphi_{, \mu}^{*} \varphi_{, \nu} g^{\mu \nu} \sqrt{-g}-\delta \xi \varphi^{*} \varphi R \sqrt{-g} .
$$

The scalar propagator comes from the Lagrangian for complex Klein-Gordon theory,

$$
\mathcal{L}_{\mathrm{CKG}}=-\varphi_{, \mu}^{*} \varphi_{, \nu} g^{\mu \nu} \sqrt{-g}
$$

It obeys the equation,

$$
\partial_{\mu}\left(\sqrt{-g} g^{\mu \nu} \partial_{\nu} i \Delta_{A}\left(x ; x^{\prime}\right)\right)=i \delta^{D}\left(x-x^{\prime}\right) .
$$

The photon propagator derives from the Lagrangian for electromagnetism,

$$
\begin{aligned}
\mathcal{L}_{\mathrm{EM}}= & -\frac{1}{4} F_{\mu \nu} F_{\rho \sigma} g^{\mu \rho} g^{\nu \sigma} \sqrt{-g} \\
= & -\frac{1}{2} A_{\mu ; \nu} A_{\rho ; \sigma} g^{\mu \rho} g^{\nu \sigma} \sqrt{-g}+\frac{1}{2} A_{\mu ; \nu} A_{\sigma ; \rho} g^{\mu \rho} g^{\nu \sigma} \sqrt{-g} \\
= & -\frac{1}{2} A_{\mu ; \nu} A_{\rho ; \sigma} g^{\mu \rho} g^{\nu \sigma} \sqrt{-g}-\frac{1}{2} A_{\mu} A_{\nu} R^{\mu \nu} \sqrt{-g}+\frac{1}{2} A_{\mu ; \nu} A_{\rho ; \sigma} g^{\mu \nu} g^{\rho \sigma} \sqrt{-g} \\
& \quad+\frac{1}{2} \partial_{\rho}\left(A_{\mu ; \nu} A_{\sigma} g^{\mu \rho} g^{\nu \sigma} \sqrt{-g}-A_{\sigma ; \mu} A_{\nu} g^{\mu \sigma} g^{\nu \rho} \sqrt{-g}\right)
\end{aligned}
$$

Our paradigm for the geometry of inflation is the open conformal coordinate patch of de Sitter,

$$
d s^{2}=a^{2}\left(-d \eta^{2}+d \vec{x} \cdot d \vec{x}\right) \quad \text { where } \quad a(\eta)=-\frac{1}{H \eta} .
$$

Note that the conformal time $\eta$ runs from $-\infty$ to zero. In this background the various metric-dependent quantities that appear in $\mathcal{L}_{\mathrm{SQED}}$ are,

$$
\begin{aligned}
& g^{\mu \nu}=a^{-2} \eta^{\mu \nu} \quad, \quad \sqrt{-g}=a^{D} \quad, \quad \Gamma_{\mu \nu}^{\rho}=H a\left(\delta_{\mu}^{\rho} \delta_{\nu}^{0}+\delta_{\nu}^{\rho} \delta_{\mu}^{0}+\delta_{0}^{\rho} \eta_{\mu \nu}\right) \\
& R=D(D-1) H^{2} \quad \text { and } \quad R^{\mu \nu}=(D-1) H^{2} a^{-2} \eta^{\mu \nu} .
\end{aligned}
$$


We shall henceforth raise and lower indicies with the Lorentz metric,

$$
\partial^{\mu} \equiv \eta^{\mu \nu} \partial_{\nu} \quad, \quad \partial^{2} \equiv \eta^{\mu \nu} \partial_{\mu} \partial_{\nu} \quad, \quad A^{\mu} \equiv \eta^{\mu \nu} A_{\nu}
$$

The special properties of our locally de Sitter background can be used to simplify the three volume terms of the electromagnetic Lagrangian (14). The first becomes,

$$
\begin{aligned}
-\frac{1}{2} A_{\mu ; \nu} A_{\rho ; \sigma} g^{\mu \rho} g^{\nu \sigma} \sqrt{-g}=-\frac{1}{2} a^{D-4}\left\{A_{\mu, \nu} A^{\mu, \nu}+2 H a A_{\mu, 0} A^{\mu}\right. \\
\left.+2 H a A_{0, \mu} A^{\mu}-2 H a A_{, \mu}^{\mu} A_{0}-2 H^{2} a^{2} A_{\mu} A^{\mu}+(D-2) H^{2} a^{2} A_{0}^{2}\right\} . \\
=-\frac{1}{2} a^{D-4} A_{\mu, \nu} A^{\mu, \nu}+\frac{1}{2}(D-1) H^{2} a^{D-2} A_{\mu} A^{\mu}+2 H a^{D-3} A_{0} A_{, \mu}^{\mu} \\
-\frac{1}{2}(3 D-8) H^{2} a^{D-2} A_{0}^{2}-\frac{1}{2}\left(H a^{D-3} A_{\mu} A^{\mu}\right)_{, 0}-\left(H a^{D-3} A_{0} A^{\mu}\right)_{, \mu} .
\end{aligned}
$$

The second volume term is simple by comparison,

$$
-\frac{1}{2} A_{\mu} A_{\nu} R^{\mu \nu} \sqrt{-g}=-\frac{1}{2}(D-1) H^{2} a^{D-2} A_{\mu} A^{\mu} .
$$

The final volume term is,

$$
\frac{1}{2}\left(A_{\mu ; \nu} g^{\mu \nu}\right)^{2} \sqrt{-g}=\frac{1}{2} a^{D-4}\left(A_{, \mu}^{\mu}\right)^{2}-(D-2) H a^{D-3} A_{0} A_{, \mu}^{\mu}+\frac{1}{2}(D-2)^{2} H^{2} a^{D-2} A_{0}^{2} .
$$

The three volume terms can be summed to give,

$$
\begin{aligned}
\mathcal{L}_{\mathrm{EM}}-(\text { Surface Term })=-\frac{1}{2} a^{D-4} A_{\mu, \nu} A^{\mu, \nu} & +\frac{1}{2}(D-4) a^{D-2} H^{2} A_{0}^{2} \\
& +\frac{1}{2} a^{D-4}\left(A_{, \mu}^{\mu}-(D-4) H a A_{0}\right)^{2} .
\end{aligned}
$$

The final expression in (22) suggests that we add the gauge fixing term [16],

$$
\mathcal{L}_{\mathrm{GF}}=-\frac{1}{2} a^{D-4}\left(A^{\mu}{ }_{, \mu}-(D-4) H a A_{0}\right)^{2} .
$$

In this gauge the photon propagator obeys the equation,

$$
\left(\partial^{\sigma} a^{D-4} \partial_{\sigma} \delta_{\mu}^{\rho}-(D-4) H^{2} a^{D-2} \delta_{\mu}^{0} \delta_{0}^{\rho}\right) i\left[{ }_{\rho} \Delta_{\nu}\right]\left(x ; x^{\prime}\right)=\eta_{\mu \nu} i \delta^{D}\left(x-x^{\prime}\right) .
$$


Because space and time components are treated differently it is useful to have expressions for the purely spatial parts of the Minkowski metric and the Kronecker delta,

$$
\bar{\eta}_{\mu \nu} \equiv \eta_{\mu \nu}+\delta_{\mu}^{0} \delta_{\nu}^{0} \quad, \quad \bar{\delta}_{\nu}^{\mu} \equiv \delta_{\nu}^{\mu}-\delta_{0}^{\mu} \delta_{\nu}^{0}
$$

Making the ansatz,

$$
i\left[{ }_{\mu} \Delta_{\nu}\right]\left(x ; x^{\prime}\right)=a a^{\prime} \bar{\eta}_{\mu \nu} i \Delta_{B}\left(x ; x^{\prime}\right)-a a^{\prime} \delta_{\mu}^{0} \delta_{\nu}^{0} i \Delta_{C}\left(x ; x^{\prime}\right) .
$$

we see that the propagators $i \Delta_{B}\left(x ; x^{\prime}\right)$ and $i \Delta_{C}\left(x ; x^{\prime}\right)$ obey the equations of scalars with various amounts of conformal coupling,

$$
\begin{aligned}
& {\left[\partial_{\mu}\left(\sqrt{-g} g^{\mu \nu} \partial_{\nu}\right)-\frac{1}{D}\left(\frac{D-2}{D-1}\right) R \sqrt{-g}\right] i \Delta_{B}\left(x ; x^{\prime}\right)=i \delta^{D}\left(x-x^{\prime}\right)} \\
& {\left[\partial_{\mu}\left(\sqrt{-g} g^{\mu \nu} \partial_{\nu}\right)-\frac{2}{D}\left(\frac{D-3}{D-1}\right) R \sqrt{-g}\right] i \Delta_{C}\left(x ; x^{\prime}\right)=i \delta^{D}\left(x-x^{\prime}\right) .}
\end{aligned}
$$

Of course the fact that we have written equations (27, 28) in an invariant form does not alter the fact that our photon propagator only applies to a locally de Sitter background.

The various propagator equations (11) and (27) 28) can be almost entirely solved in terms of the following function of the invariant length $\ell\left(x ; x^{\prime}\right)$ between $x^{\mu}$ and $x^{\prime \mu}$,

$$
y\left(x ; x^{\prime}\right) \equiv 4 \sin ^{2}\left(\frac{1}{2} H \ell\left(x ; x^{\prime}\right)\right)=a a^{\prime} H^{2}\left(\left\|\vec{x}-\vec{x}^{\prime}\right\|^{2}-\left(\left|\eta-\eta^{\prime}\right|-i \delta\right)^{2}\right) .
$$

The most singular part of each propagator is the same as the propagator for a massless, conformally coupled scalar [19],

$$
i \Delta_{\mathrm{cf}}\left(x ; x^{\prime}\right)=\frac{H^{D-2}}{(4 \pi)^{\frac{D}{2}}} \Gamma\left(\frac{D}{2}-1\right)\left(\frac{4}{y}\right)^{\frac{D}{2}-1} .
$$

It has long been known that no de Sitter invariant solution exists for the $A$ type propagator [17]. It is natural to imagine our locally de Sitter background as one of the larger class of conformally flat geometries but with arbitrary time dependence in the scale factor. In that case the relevant symmetry to 
preserve is spatial homogeneity and isotropy, which is known as the "E(3)" vacuum [20]. The minimal solution for this is [21, 22,

$$
\begin{aligned}
& i \Delta_{A}\left(x ; x^{\prime}\right)=i \Delta_{\mathrm{cf}}\left(x ; x^{\prime}\right) \\
& +\frac{H^{D-2}}{(4 \pi)^{\frac{D}{2}}} \frac{\Gamma(D-1)}{\Gamma\left(\frac{D}{2}\right)}\left\{\frac{D}{D-4} \frac{\Gamma^{2}\left(\frac{D}{2}\right)}{\Gamma(D-1)}\left(\frac{4}{y}\right)^{\frac{D}{2}-2}-\pi \cot \left(\frac{\pi}{2} D\right)+\ln \left(a a^{\prime}\right)\right\} \\
& +\frac{H^{D-2}}{(4 \pi)^{\frac{D}{2}}} \sum_{n=1}^{\infty}\left\{\frac{1}{n} \frac{\Gamma(n+D-1)}{\Gamma\left(n+\frac{D}{2}\right)}\left(\frac{y}{4}\right)^{n}-\frac{1}{n-\frac{D}{2}+2} \frac{\Gamma\left(n+\frac{D}{2}+1\right)}{\Gamma(n+2)}\left(\frac{y}{4}\right)^{n-\frac{D}{2}+2}\right\}
\end{aligned}
$$

On the other hand, the $B$-type and $C$-type propagators possess unique, de Sitter invariant solutions [23, 24],

$$
\begin{aligned}
& i \Delta_{B}\left(x ; x^{\prime}\right)=\frac{H^{D-2}}{(4 \pi)^{\frac{D}{2}}} \frac{\Gamma(D-2) \Gamma(1)}{\Gamma\left(\frac{D}{2}\right)}{ }_{2} F_{1}\left(D-2,1 ; \frac{D}{2} ; 1-\frac{y}{4}\right), \\
& i \Delta_{C}\left(x ; x^{\prime}\right)=\frac{H^{D-2}}{(4 \pi)^{\frac{D}{2}}} \frac{\Gamma(D-3) \Gamma(2)}{\Gamma\left(\frac{D}{2}\right)}{ }_{2} F_{1}\left(D-3,2 ; \frac{D}{2} ; 1-\frac{y}{4}\right) .
\end{aligned}
$$

One can note the progression in the coefficients of the conformal coupling terms in the equations for $i \Delta_{A}, i \Delta_{B}$ and $i \Delta_{C}$,

$$
-\frac{0}{D}\left(\frac{D-1}{D-1}\right) R \sqrt{-g} \longrightarrow-\frac{1}{D}\left(\frac{D-2}{D-1}\right) R \sqrt{-g} \longrightarrow-\frac{2}{D}\left(\frac{D-3}{D-1}\right) R \sqrt{-g} .
$$

The same progression is evident in the $B$-type and $C$-type solutions (32) 33 ). Were it extended to the $A$-type solution the progression would give,

$$
\frac{H^{D-2}}{(4 \pi)^{\frac{D}{2}}} \frac{\Gamma(D-1) \Gamma(0)}{\Gamma\left(\frac{D}{2}\right)}{ }_{2} F_{1}\left(D-1,0 ; \frac{D}{2} ; 1-\frac{y}{4}\right) .
$$

However, expression (35) is singular, which reflects the incompatibility of assuming a de Sitter invariant solution.

Rather than trying to write $i \Delta_{A}\left(x ; x^{\prime}\right)$ in terms of hypergeometric functions it is actually more effective to expand $i \Delta_{B}\left(x ; x^{\prime}\right)$ and $i \Delta_{C}\left(x ; x^{\prime}\right)$ in the same form as $i \Delta_{A}\left(x ; x^{\prime}\right)$,

$$
\begin{array}{r}
i \Delta_{B}\left(x ; x^{\prime}\right)=i \Delta_{\mathrm{cf}}\left(x ; x^{\prime}\right)-\frac{H^{D-2}}{(4 \pi)^{\frac{D}{2}}} \sum_{n=0}^{\infty}\left\{\frac{\Gamma(n+D-2)}{\Gamma\left(n+\frac{D}{2}\right)}\left(\frac{y}{4}\right)^{n}\right. \\
\left.-\frac{\Gamma\left(n+\frac{D}{2}\right)}{\Gamma(n+2)}\left(\frac{y}{4}\right)^{n-\frac{D}{2}+2}\right\},
\end{array}
$$




$$
\begin{aligned}
& i \Delta_{C}\left(x ; x^{\prime}\right)=i \Delta_{\mathrm{cf}}\left(x ; x^{\prime}\right)+ \frac{H^{D-2}}{(4 \pi)^{\frac{D}{2}}} \sum_{n=0}^{\infty}\left\{(n+1) \frac{\Gamma(n+D-3)}{\Gamma\left(n+\frac{D}{2}\right)}\left(\frac{y}{4}\right)^{n}\right. \\
&\left.-\left(n-\frac{D}{2}+3\right) \frac{\Gamma\left(n+\frac{D}{2}-1\right)}{\Gamma(n+2)}\left(\frac{y}{4}\right)^{n-\frac{D}{2}+2}\right\} .
\end{aligned}
$$

These expressions tend to intimidate but it will be seen that they are quite simple to use on account of the facts that the infinite sums vanish for $D=4$ and each term goes like a positive power of $y\left(x ; x^{\prime}\right)$. Hence the infinite sums can only contribute when multiplied by a term which becomes sufficiently singular upon coincidence. Note also that the $B$-type and $C$-type propagators degenerate to $i \Delta_{\text {cf }}$ in $D=4$, so the photon propagator is the same for $D=4$ as it is in flat space! This is the gauge that takes full advantage of the fact that electromagnetism is conformally invariant for $D=4$.

\section{$3 \quad M^{2}\left(x ; x^{\prime}\right)$ in the Simplest Gauge}

The scalar self-mass-squared receives one loop contributions from a single 4-point interaction (8), from a product of two 3-point interactions (77), and from the renormalization counterterms (9). One sub-section is devoted to the evaluation of each sort of contribution. By far the most difficult is the contribution from two 3-point interactions. That requires four separate parts!

\subsection{Contributions from the 4-Point Interaction}

The 4-point interaction (8) gives rise to a diagram with the topology depicted in Fig. 1. Its contribution is,

$$
\left.-i M_{4 \mathrm{pt}}^{2}\left(x ; x^{\prime}\right)=-i e^{2} \sqrt{-g} g^{\mu \nu} i{ }_{\mu} \Delta_{\nu}\right]\left(x ; x^{\prime}\right) \delta^{D}\left(x-x^{\prime}\right) .
$$

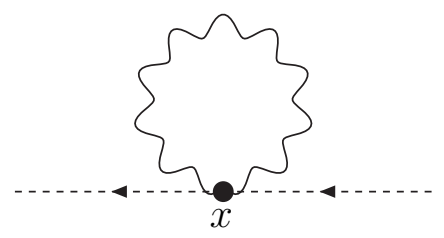

Fig. 1: Contribution from the 4-point interaction. 
We obviously require the coincidence limits of the $B$-type and $C$-type propagators,

$$
\begin{aligned}
& \lim _{x^{\prime} \rightarrow x} i \Delta_{B}\left(x ; x^{\prime}\right)=\frac{H^{D-2}}{(4 \pi)^{\frac{D}{2}}} \frac{\Gamma(D-1)}{\Gamma\left(\frac{D}{2}\right)} \times-\frac{1}{D-2} \\
& \lim _{x^{\prime} \rightarrow x} i \Delta_{C}\left(x ; x^{\prime}\right)=\frac{H^{D-2}}{(4 \pi)^{\frac{D}{2}}} \frac{\Gamma(D-1)}{\Gamma\left(\frac{D}{2}\right)} \times \frac{1}{(D-2)(D-3)} .
\end{aligned}
$$

It follows that our result for the 4-point contribution is completely finite in $D=4$ dimensions,

$$
\begin{aligned}
& -i M_{4 \mathrm{pt}}^{2}\left(x ; x^{\prime}\right) \\
& =\frac{i e^{2} H^{D-2} a^{D}}{(4 \pi)^{\frac{D}{2}}} \frac{\Gamma(D-1)}{\Gamma\left(\frac{D}{2}\right)}\left\{\left(\frac{D-1}{D-2}\right)-\frac{1}{(D-2)(D-3)}\right\} \delta^{D}\left(x-x^{\prime}\right), \\
& \quad \longrightarrow \frac{i e^{2} H^{2}}{8 \pi^{2}} a^{4} \delta^{4}\left(x-x^{\prime}\right) .
\end{aligned}
$$

\subsection{Contributions from Two 3-Point Interactions}

The 3-point interaction (7) gives rise to a diagram with the topology depicted in Fig. 2. Its contribution is,

$$
\begin{aligned}
-i M_{3 \mathrm{pt}}^{2}\left(x ; x^{\prime}\right)= & -e^{2} \sqrt{-g} g^{\mu \nu} \sqrt{-g^{\prime}} g^{\prime \rho \sigma} i\left[{ }_{\mu} \Delta_{\rho}\right]\left(x ; x^{\prime}\right) \partial_{\nu} \partial_{\sigma}^{\prime} i \Delta_{A}\left(x ; x^{\prime}\right) \\
& \left.-e^{2} \partial_{\nu}\left[\sqrt{-g} g^{\mu \nu} \sqrt{-g^{\prime}} g^{\prime \rho \sigma} i{ }_{\mu} \Delta_{\rho}\right]\left(x ; x^{\prime}\right) \partial_{\sigma}^{\prime} i \Delta_{A}\left(x ; x^{\prime}\right)\right] \\
& \left.-e^{2} \partial_{\sigma}^{\prime}\left[\sqrt{-g} g^{\mu \nu} \sqrt{-g^{\prime}} g^{\prime \rho \sigma} i{ }_{\mu} \Delta_{\rho}\right]\left(x ; x^{\prime}\right) \partial_{\nu} i \Delta_{A}\left(x ; x^{\prime}\right)\right] \\
& -e^{2} \partial_{\nu} \partial_{\sigma}^{\prime}\left[\sqrt{-g} g^{\mu \nu} \sqrt{-g^{\prime}} g^{\prime \rho \sigma} i\left[{ }_{\mu} \Delta_{\rho}\right]\left(x ; x^{\prime}\right) i \Delta_{A}\left(x ; x^{\prime}\right)\right] .
\end{aligned}
$$

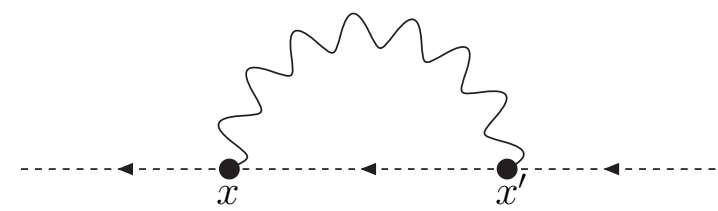

Fig. 2: Contribution from two 3-point interactions.

The key to an efficient calculation is that the most divergent term for each of the three scalar propagators is the conformal propagator,

$$
i \Delta_{I}\left(x ; x^{\prime}\right)=i \Delta_{\mathrm{cf}}\left(x ; x^{\prime}\right)+i \delta \Delta_{I}\left(x ; x^{\prime}\right) .
$$


Hence the most singular part of the photon propagator has a simple tensor structure,

$$
i\left[{ }_{\mu} \Delta_{\nu}\right]\left(x ; x^{\prime}\right)=a a^{\prime} \eta_{\mu \nu} i \Delta_{\mathrm{cf}}\left(x ; x^{\prime}\right)+a a^{\prime} \bar{\eta}_{\mu \nu} i \delta \Delta_{B}\left(x ; x^{\prime}\right)-a a^{\prime} \delta_{\mu}^{0} \delta_{\nu}^{0} i \delta \Delta_{C}\left(x ; x^{\prime}\right) .
$$

Now recall that only the $n=0$ term of $i \delta \Delta_{A}$ fails to vanish in $D=4$ dimensions. Further, the $n$-th term in each $i \delta \Delta_{I}$ goes like $y^{n}$, so these terms can only contribute when multiplied by something sufficiently singular from another propagator. Because this diagram is at worst quadratically divergent - and that only from two factors of $i \Delta_{\mathrm{cf}}$ - no more than one factor of $i \delta \Delta_{I}$ can give a nonzero contribution, and only from the $n=0$ terms. We therefore partition this subsection into computing the respective contributions from when both the scalar and the photon propagators provide $i \Delta_{\text {cf }}$, from when the scalar provides the $n=0$ term of $i \delta \Delta_{A}$ times $i \Delta_{\text {cf }}$ from the photon propagator, and from when the photon provides either $i \delta \Delta_{B}$ or $i \delta \Delta_{C}$ times $i \Delta_{\text {cf }}$ from the scalar propagator.

\subsubsection{Contributions from $i \Delta_{\mathrm{cf}} \times i \Delta_{\mathrm{cf}}$}

The tensor structure of the conformal part of the photon propagator gives a simple result for the contractions in (43),

$$
\begin{array}{r}
-i M_{\mathrm{cf}}^{2}\left(x ; x^{\prime}\right)=-4 e^{2}\left(a a^{\prime}\right)^{D-1} i \Delta_{\mathrm{cf}} \partial \cdot \partial^{\prime} i \Delta_{\mathrm{cf}}-2 e^{2} a^{D-1} \partial^{\mu}\left[a^{D-1} i \Delta_{\mathrm{cf}}\right] \partial_{\mu}^{\prime} i \Delta_{\mathrm{cf}} \\
-2 e^{2} a^{D-1} \partial^{\prime \mu}\left[a^{D-1} i \Delta_{\mathrm{cf}}\right] \partial_{\mu} i \Delta_{\mathrm{cf}}-e^{2} \partial \cdot \partial^{\prime}\left[\left(a a^{\prime}\right)^{D-1} i \Delta_{\mathrm{cf}}\right] i \Delta_{\mathrm{cf}} .
\end{array}
$$

We next substitute the explicit form of the conformal propagator,

$$
i \Delta_{\mathrm{cf}}\left(x ; x^{\prime}\right)=\frac{\Gamma\left(\frac{D}{2}-1\right)}{4 \pi^{\frac{D}{2}}} \frac{\left(a a^{\prime}\right)^{1-\frac{D}{2}}}{\Delta x^{D-2}} .
$$

That brings the result to form,

$$
\begin{array}{r}
-i M_{\mathrm{cf}}^{2}\left(x ; x^{\prime}\right)=-\frac{e^{2} \Gamma^{2}\left(\frac{D}{2}-1\right)}{16 \pi^{D}}\left\{\frac{4\left(a a^{\prime}\right)^{\frac{D}{2}}}{\Delta x^{D-2}} \partial \cdot \partial^{\prime}\left[\frac{\left(a a^{\prime}\right)^{1-\frac{D}{2}}}{\Delta x^{D-2}}\right]\right. \\
+\frac{2 a^{\prime \frac{D}{2}}}{a^{\frac{D}{2}-1}} \partial^{\mu}\left[\frac{a^{\frac{D}{2}}}{\Delta x^{D-2}}\right] \partial_{\mu}^{\prime}\left[\frac{a^{\prime 1-\frac{D}{2}}}{\Delta x^{D-2}}\right]+\frac{2 a^{\frac{D}{2}}}{a^{\prime \frac{D}{2}-1}} \partial^{\prime \mu}\left[\frac{a^{\prime \frac{D}{2}}}{\Delta x^{D-2}}\right] \partial_{\mu}\left[\frac{a^{1-\frac{D}{2}}}{\Delta x^{D-2}}\right] \\
\left.+\frac{\left(a a^{\prime}\right)^{1-\frac{D}{2}}}{\Delta x^{D-2}} \partial \cdot \partial^{\prime}\left[\frac{\left(a a^{\prime}\right)^{\frac{D}{2}}}{\Delta x^{D-2}}\right]\right\} .
\end{array}
$$


Acting the derivatives is facilitated by the simple identities,

$$
\begin{aligned}
& \partial_{\mu} a^{p}=a^{p}\left\{\partial_{\mu}+p H a \delta_{\mu}^{0}\right\}, \\
& \partial \cdot \partial^{\prime}\left[\frac{\left(a a^{\prime}\right)^{p}}{\Delta x^{D-2}}\right]=\left(a a^{\prime}\right)^{p+1}\left\{\frac{(D-2) p H^{2} \Delta \eta^{2}}{\Delta x^{D}}-\frac{p^{2} H^{2}}{\Delta x^{D-2}}-\frac{i}{a^{D}} \delta^{D}\left(x-x^{\prime}\right)\right\}, \\
& \partial^{\mu}\left[\frac{a^{p}}{\Delta x^{D-2}}\right] \partial_{\mu}^{\prime}\left[\frac{a^{\prime q}}{\Delta x^{D-2}}\right]=a^{p} a^{\prime q}\left\{-\frac{(D-2)^{2}}{\Delta x^{2 D-2}}-\frac{(D-2) q a^{\prime} H \Delta \eta}{\Delta x^{2 D-2}}\right. \\
& \left.+\frac{(D-2) p a H \Delta \eta}{\Delta x^{2 D-2}}-\frac{p q H^{2} a a^{\prime}}{\Delta x^{2 D-4}}\right\} .
\end{aligned}
$$

The result is,

$$
\begin{aligned}
-i M_{\mathrm{cf}}^{2}\left(x ; x^{\prime}\right)=\frac{e^{2} \Gamma^{2}\left(\frac{D}{2}-1\right)}{16 \pi^{D}}\left\{\frac{4(D-2)^{2} a a^{\prime}}{\Delta x^{2 D-2}}\right. & \\
& \left.+\frac{\frac{3}{2}(D-4)(D-2) a^{2} a^{\prime 2} H^{2} \Delta \eta^{2}}{\Delta x^{2 D-2}}+\frac{\frac{1}{4}(D-4)^{2} H^{2} a^{2} a^{\prime 2}}{\Delta x^{2 D-4}}\right\} .
\end{aligned}
$$

At this point it is useful to recall that the physics of $M^{2}\left(x ; x^{\prime}\right)$ is inferred by integrating it up against a smooth function in the linearized effective field equation (11). Although each of the three terms in (52) is singular at $x^{\prime \mu}=x^{\mu}$ (and hence $\Delta x^{2}=0$ ), they can each be expressed as the derivatives (with respect to $x^{\mu}$ ) of functions that are integrable in $D=4$ dimensions. Identities which facilitate this are,

$$
\begin{aligned}
\frac{1}{\Delta x^{2 D-4}} & =\frac{\partial^{2}}{2(D-3)(D-4)}\left(\frac{1}{\Delta x^{2 D-6}}\right) \\
\frac{1}{\Delta x^{2 D-2}}= & \frac{\partial^{4}}{4(D-2)^{2}(D-3)(D-4)}\left(\frac{1}{\left.\Delta x^{2 D-6}\right)}\right. \\
\frac{\Delta \eta^{2}}{\Delta x^{2 D-2}=} & \frac{\partial_{0}^{2}}{4(D-2)(D-3)}\left(\frac{1}{\left.\Delta x^{2 D-6}\right)}\right. \\
& \quad-\frac{\partial^{2}}{4(D-2)(D-3)(D-4)}\left(\frac{1}{\Delta x^{2 D-6}}\right) .
\end{aligned}
$$

The resulting expression for the conformal contributions to the self-masssquared is,

$$
\begin{aligned}
-i M_{\mathrm{cf}}^{2}\left(x ; x^{\prime}\right)=\frac{e^{2} \Gamma^{2}\left(\frac{D}{2}-1\right)}{16 \pi^{D}(D-3)}\left\{\frac{a a^{\prime} \partial^{4}}{D-4}+\frac{3}{8}(D-4) a^{2} a^{\prime 2} H^{2} \partial_{0}^{2}\right. \\
\left.+\frac{1}{8}(D-7) H^{2} a^{2} a^{\prime 2} \partial^{2}\right\}\left(\frac{1}{\Delta x^{2 D-6}}\right) .
\end{aligned}
$$


All of the expressions in (56) are integrable with respect to $x^{\prime \mu}$ in the effective field equation (11). We could take $D=4$ at this stage were it not for the explicit factor of $1 /(D-4)$ in the first term. This is an ultraviolet divergence, however, it is not yet in the correct form to be removed by a local counterterm. We can achieve this form by employing the identity,

$$
\partial^{2}\left(\frac{1}{\Delta x^{D-2}}\right)=\frac{i 4 \pi^{\frac{D}{2}}}{\Gamma\left(\frac{D}{2}-1\right)} \delta^{D}\left(x-x^{\prime}\right) .
$$

The procedure is to first add zero to the basic divergence,

$\frac{\partial^{2}}{D-4}\left(\frac{1}{\Delta x^{2 D-6}}\right)=\frac{\partial^{2}}{D-4}\left(\frac{1}{\Delta x^{2 D-6}}-\frac{\mu^{D-4}}{\Delta x^{D-2}}\right)+\frac{\mu^{D-4}}{D-4} \times \frac{i 4 \pi^{\frac{D}{2}}}{\Gamma\left(\frac{D}{2}-1\right)} \delta^{D}\left(x-x^{\prime}\right)$.

The parenthesized nonlocal term is not only integrable in $D=4$, it also vanishes in that dimension,

$$
\begin{aligned}
\frac{1}{\Delta x^{2 D-6}}-\frac{\mu^{D-4}}{\Delta x^{D-2}} & =\frac{\mu^{2 D-8}}{\Delta x^{2}}\left\{\left(\frac{1}{\mu^{2} \Delta x^{2}}\right)^{D-4}-\left(\frac{1}{\mu^{2} \Delta x^{2}}\right)^{\frac{D}{2}-2}\right\}, \\
& =-\frac{1}{2}(D-4) \frac{\ln \left(\mu^{2} \Delta x^{2}\right)}{\Delta x^{2}}+O\left((D-4)^{2}\right) .
\end{aligned}
$$

We can therefore segregate the basic divergence on a local term — which can be absorbed into a counterterm - and take the limit $D=4$ on the finite, nonlocal term,

$$
\frac{\partial^{2}}{D-4}\left(\frac{1}{\Delta x^{2 D-6}}\right)=\frac{i 4 \pi^{\frac{D}{2}} \mu^{D-4}}{\Gamma\left(\frac{D}{2}-1\right)} \frac{\delta^{D}\left(x-x^{\prime}\right)}{D-4}-\frac{1}{2} \partial^{2}\left(\frac{\ln \left(\mu^{2} \Delta x^{2}\right)}{\Delta x^{2}}\right)+O(D-4) .
$$

Employing this in (56) and taking the other terms to $D=4$ gives,

$$
\begin{aligned}
-i M_{\mathrm{cf}}^{2}(x ; & \left.x^{\prime}\right)=\frac{i e^{2} \mu^{D-4}}{4 \pi^{\frac{D}{2}}} \frac{\Gamma\left(\frac{D}{2}-1\right)}{(D-3)(D-4)} a a^{\prime} \partial^{2} \delta^{D}\left(x-x^{\prime}\right) \\
- & \frac{i 3 e^{2} H^{2}}{2^{5} \pi^{2}} a^{4} \delta^{4}\left(x-x^{\prime}\right)-\frac{e^{2}}{2^{5} \pi^{4}} a a^{\prime} \partial^{4}\left(\frac{\ln \left(\mu^{2} \Delta x^{2}\right)}{\Delta x^{2}}\right)+O(D-4) .
\end{aligned}
$$

\subsubsection{Contributions from $i \Delta_{\mathrm{cf}} \times i \delta \Delta_{A}$}

Recall that the full scalar propagator is $i \Delta_{A}\left(x ; x^{\prime}\right)=i \Delta_{\text {cf }}\left(x ; x^{\prime}\right)+i \delta \Delta_{A}\left(x ; x^{\prime}\right)$. We have just computed the result of keeping only the conformal parts of the 
photon and scalar propagators. Using just the conformal part of the photon propagator and setting the scalar propagator to $i \delta \Delta_{A}$ gives,

$$
\begin{array}{r}
-i M_{\delta A}^{2}\left(x ; x^{\prime}\right)=-e^{2}\left(a a^{\prime}\right)^{D-1} i \Delta_{\mathrm{cf}} \partial \cdot \partial^{\prime} i \delta \Delta_{A}-e^{2} a^{D-1} \partial^{\mu}\left[a^{D-1} i \Delta_{\mathrm{cf}} \partial_{\mu}^{\prime} i \delta \Delta_{A}\right] \\
-e^{2} a^{D-1} \partial^{\prime \mu}\left[a^{D-1} i \Delta_{\mathrm{cf}} \partial_{\mu} i \delta \Delta_{A}\right]-e^{2} \partial \cdot \partial^{\prime}\left[\left(a a^{\prime}\right)^{D-1} i \Delta_{\mathrm{cf}} i \delta \Delta_{A}\right] .
\end{array}
$$

It is useful to separately consider the reduction of each of the four terms on the right-hand side of (63), labeling them "1", "2", "3" and "4", respectively.

We can read off $i \delta \Delta_{A}$ from the $A$-type propagator (31),

$$
\begin{aligned}
& i \delta \Delta_{A}\left(x ; x^{\prime}\right)= \\
& \quad \frac{H^{2}}{16 \pi^{\frac{D}{2}}} \frac{\Gamma\left(\frac{D}{2}+1\right)}{\frac{D}{2}-2} \frac{\left(a a^{\prime}\right)^{2-\frac{D}{2}}}{\Delta x^{D-4}}+\frac{H^{D-2}}{(4 \pi)^{\frac{D}{2}}} \frac{\Gamma(D-1)}{\Gamma\left(\frac{D}{2}\right)}\left\{-\pi \cot \left(\frac{\pi}{2} D\right)+\ln \left(a a^{\prime}\right)\right\} \\
& +\frac{H^{D-2}}{(4 \pi)^{\frac{D}{2}}} \sum_{n=1}^{\infty}\left\{\frac{1}{n} \frac{\Gamma(n+D-1)}{\Gamma\left(n+\frac{D}{2}\right)}\left(\frac{y}{4}\right)^{n}-\frac{1}{n-\frac{D}{2}+2} \frac{\Gamma\left(n+\frac{D}{2}+1\right)}{\Gamma(n+2)}\left(\frac{y}{4}\right)^{n-\frac{D}{2}+2}\right\} .(
\end{aligned}
$$

In $D=4$ the most singular contributions to (63) have the form, $i \delta \Delta_{A} / \Delta x^{4}$. Because the infinite series terms in (64) go like positive powers of $\Delta x^{2}$ these terms make integrable contributions to (63). We can therefore take $D=4$, at which point we see that all the infinite series terms drop. It is sometimes also useful to note that the $D=4$ limit is,

$$
i \delta \Delta_{A}\left(x ; x^{\prime}\right)=-\frac{H^{2}}{8 \pi^{2}} \ln \left(\frac{\sqrt{e}}{4} H^{2} \Delta x^{2}\right)+O(D-4) .
$$

Note that the factor of $e$ in this expression is the base of the natural logarithm rather than the electromagnetic coupling constant! The distinction should always be clear from context.

We begin the reduction of the first term in (63) by substituting the conformal propagator,

$$
\begin{aligned}
-i M_{\delta A_{1}}^{2}\left(x ; x^{\prime}\right) & \equiv-e^{2}\left(a a^{\prime}\right)^{D-1} i \Delta_{\mathrm{cf}}\left(x ; x^{\prime}\right) \partial \cdot \partial^{\prime} i \delta \Delta_{A}\left(x ; x^{\prime}\right), \\
& =-\frac{e^{2} \Gamma\left(\frac{D}{2}-1\right)}{4 \pi^{\frac{D}{2}}} \frac{\left(a a^{\prime}\right)^{\frac{D}{2}}}{\Delta x^{D-4}} \partial \cdot \partial^{\prime} i \delta \Delta_{A}\left(x ; x^{\prime}\right) .
\end{aligned}
$$

Only the first term of (64) survives when acted upon by $\partial \cdot \partial^{\prime}$, 


$$
\begin{array}{r}
-i M_{\delta A_{1}}^{2}\left(x ; x^{\prime}\right) \\
=-\frac{e^{2} H^{2}}{2^{6} \pi^{D}} \frac{\Gamma\left(\frac{D}{2}-1\right) \Gamma\left(\frac{D}{2}+1\right)}{\frac{D}{2}-2} \frac{\left(a a^{\prime}\right)^{\frac{D}{2}}}{\Delta x^{D-2}} \partial \cdot \partial^{\prime}\left[\frac{\left(a a^{\prime}\right)^{2-\frac{D}{2}}}{\Delta x^{D-4}}\right]+O(D-4), \\
=\frac{e^{2} H^{2}}{2^{6} \pi^{D}} \Gamma\left(\frac{D}{2}-1\right) \Gamma\left(\frac{D}{2}+1\right)\left(a a^{\prime}\right)^{2}\left\{-\frac{4}{\Delta x^{2 D-4}+\frac{(D-4) a a^{\prime} H^{2} \Delta \eta^{2}}{\Delta x^{2 D-4}}}\right. \\
\left.+\frac{(D-4) a a^{\prime} H^{2}}{2 \Delta x^{2 D-6}}\right\}+O(D-4) .
\end{array}
$$

The first term in this expression is of a type we have considered in the previous sub-section. On the other hand, the last two terms are integrable, so they give zero contribution on account of the factors of $D-4$. The final result is,

$$
\begin{aligned}
-i M_{\delta A_{1}}^{2}\left(x ; x^{\prime}\right)=-\frac{i e^{2} H^{2} \mu^{D-4}}{8 \pi^{\frac{D}{2}}} & \frac{\Gamma\left(\frac{D}{2}+1\right)}{(D-3)(D-4)} a^{4} \delta^{D}\left(x-x^{\prime}\right) \\
+ & \frac{e^{2} H^{2}}{2^{5} \pi^{4}}\left(a a^{\prime}\right)^{2} \partial^{2}\left(\frac{\ln \left(\mu^{2} \Delta x^{2}\right)}{\Delta x^{2}}\right)+O(D-4) .
\end{aligned}
$$

The second term in (63) is,

$$
\begin{aligned}
-i M_{\delta A_{2}}^{2}\left(x ; x^{\prime}\right) \equiv-e^{2} a^{\prime D-1} \partial^{\mu}\left[a^{D-1} i \Delta_{\mathrm{cf}}\left(x ; x^{\prime}\right) \partial_{\mu}^{\prime} i \delta \Delta_{A}\left(x ; x^{\prime}\right)\right], \\
=-\frac{e^{2} \Gamma\left(\frac{D}{2}-1\right)}{4 \pi^{\frac{D}{2}}}\left(a a^{\prime}\right)^{\frac{D}{2}}\left\{\partial^{\mu}\left[\frac{\partial_{\mu}^{\prime} i \delta \Delta_{A}}{\Delta x^{D-2}}\right]-\frac{D H a}{2 \Delta x^{D-2}} \partial_{0}^{\prime} i \delta \Delta_{A}\right\} .
\end{aligned}
$$

The derivative of $i \delta \Delta_{A}\left(x ; x^{\prime}\right)$ is,

$$
\begin{aligned}
\partial_{\mu}^{\prime} i \delta \Delta_{A}\left(x ; x^{\prime}\right)=\frac{H^{2}}{16 \pi^{\frac{D}{2}}} \Gamma\left(\frac{D}{2}+1\right) & \left(a a^{\prime}\right)^{2-\frac{D}{2}}\left[\frac{2 \Delta x_{\mu}}{\Delta x^{D-2}}-\frac{H a^{\prime} \delta_{\mu}^{0}}{\Delta x^{D-4}}\right] \\
+ & \frac{H^{D-2}}{2^{D} \pi^{\frac{D}{2}}} \frac{\Gamma(D-1)}{\Gamma\left(\frac{D}{2}\right)} H a^{\prime} \delta_{\mu}^{0}+O(D-4) .
\end{aligned}
$$

Combining the two results gives,

$$
\begin{aligned}
&-i M_{\delta A_{2}}^{2}\left(x ; x^{\prime}\right)=\frac{e^{2} H^{2}}{2^{6} \pi^{D}} \Gamma\left(\frac{D}{2}-1\right) \Gamma\left(\frac{D}{2}+1\right) \\
& \times\left(a a^{\prime}\right)^{2}\left\{\frac{2(D-4)}{\Delta x^{2 D-4}}-\frac{2(D-3) a^{\prime} H \Delta \eta}{\Delta x^{2 D-4}}-\frac{4 a H \Delta \eta}{\Delta x^{2 D-4}}-\frac{2 a a^{\prime} H^{2}}{\Delta x^{2 D-6}}\right\} \\
&+\frac{e^{2} H^{D-2}}{2^{D+2} \pi^{D}} \Gamma(D-1)\left(a a^{\prime}\right)^{\frac{D}{2}}\left\{\frac{2 a^{\prime} H \Delta \eta}{\Delta x^{D}}+\frac{D a a^{\prime} H^{2}}{(D-2) \Delta x^{D-2}}\right\}+O(D-4) .
\end{aligned}
$$


Of course the third term in (63) follows from the second just by interchanging $x^{\mu}$ and $x^{\prime \mu}$,

$$
\begin{aligned}
&-i M_{\delta A_{3}}^{2}(\left.; x^{\prime}\right)=\frac{e^{2} H^{2}}{2^{6} \pi^{D}} \Gamma\left(\frac{D}{2}-1\right) \Gamma\left(\frac{D}{2}+1\right) \\
& \times\left(a a^{\prime}\right)^{2}\left\{\frac{2(D-4)}{\Delta x^{2 D-4}}+\frac{2(D-3) a H \Delta \eta}{\Delta x^{2 D-4}}+\frac{4 a^{\prime} H \Delta \eta}{\Delta x^{2 D-4}}-\frac{2 a a^{\prime} H^{2}}{\Delta x^{2 D-6}}\right\} \\
&+\frac{e^{2} H^{D-2}}{2^{D+2} \pi^{D}} \Gamma(D-1)\left(a a^{\prime}\right)^{\frac{D}{2}}\left\{-\frac{2 a H \Delta \eta}{\Delta x^{D}}+\frac{D a a^{\prime} H^{2}}{(D-2) \Delta x^{D-2}}\right\}+O(D-4) .
\end{aligned}
$$

The fact that $a-a^{\prime}=a a^{\prime} H \Delta \eta$ allows us to usefully combine (174) and (75),

$$
\begin{aligned}
& -i M_{\delta A_{2+3}}^{2}\left(x ; x^{\prime}\right)=\frac{e^{2} H^{2}}{2^{6} \pi^{D}} \Gamma\left(\frac{D}{2}-1\right) \Gamma\left(\frac{D}{2}+1\right) \\
& \quad \times\left(a a^{\prime}\right)^{2}\left\{\frac{4(D-4)}{\Delta x^{2 D-4}}+\frac{2(D-5) a a^{\prime} H^{2} \Delta \eta^{2}}{\Delta x^{2 D-4}}-\frac{4 a a^{\prime} H^{2}}{\Delta x^{2 D-6}}\right\} \\
& +\frac{e^{2} H^{D-2}}{2^{D+2} \pi^{D}} \Gamma(D-1)\left(a a^{\prime}\right)^{\frac{D}{2}+1}\left\{-\frac{2 H^{2} \Delta \eta^{2}}{\Delta x^{D}}+\frac{2 D H^{2}}{(D-2) \Delta x^{D-2}}\right\}+O(D-4) .
\end{aligned}
$$

Only the first term is not immediately integrable, and the explicit factor of $D-4$ it bears results in a finite, local contribution from it,

$$
\frac{4(D-4)}{\Delta x^{2 D-4}}=\frac{2 \partial^{2}}{D-3}\left(\frac{1}{\Delta x^{2 D-6}}\right)=i 8 \pi^{2} \delta^{4}\left(x-x^{\prime}\right)+O(D-4) .
$$

Hence the second and third terms in (63) make a completely finite contribution,

$$
-i M_{\delta A_{2+3}}^{2}\left(x ; x^{\prime}\right)=\frac{i e^{2} H^{2}}{8 \pi^{2}} a^{4} \delta^{4}\left(x-x^{\prime}\right)+\frac{e^{2} H^{4}}{2^{6} \pi^{4}}\left(a a^{\prime}\right)^{3}\left(2 \partial_{0}^{2}+\partial^{2}\right) \ln \left(\Delta x^{2}\right)+O(D-4) .
$$

The final term in (63) has the simplest reduction and the most interesting eventual contribution. Its reduction is accomplished by simply refraining from acting the external derivatives and instead taking the limit $D=4$ on the integrable expression inside the square brackets,

$$
\begin{gathered}
-i M_{\delta A_{4}}^{2}\left(x ; x^{\prime}\right) \equiv-e^{2} \partial \cdot \partial^{\prime}\left[\left(a a^{\prime}\right)^{D-1} i \Delta_{\mathrm{cf}}\left(x ; x^{\prime}\right) i \delta \Delta_{A}\left(x ; x^{\prime}\right)\right], \\
=\frac{e^{2} H^{2}}{2^{5} \pi^{4}} \partial \cdot \partial^{\prime}\left\{\frac{\left(a a^{\prime}\right)^{2}}{\Delta x^{2}} \ln \left(\frac{\sqrt{e}}{4} H^{2} \Delta x^{2}\right)\right\}+O(D-4),
\end{gathered}
$$




$$
\begin{array}{r}
=-\frac{e^{2} H^{2}}{2^{5} \pi^{4}}\left(a a^{\prime}\right)^{2} \partial^{2}\left\{\frac{\ln \left(\frac{\sqrt{e}}{4} H^{2} \Delta x^{2}\right)}{\Delta x^{2}}\right\}-\frac{e^{2} H^{4}}{2^{6} \pi^{4}}\left(a a^{\prime}\right)^{3}\left\{\partial_{0}^{2}\left[\ln ^{2}\left(\frac{\sqrt{e}}{4} H^{2} \Delta x^{2}\right)\right]\right. \\
\left.+\frac{3}{2} \partial^{2}\left[\ln ^{2}\left(\frac{\sqrt{e}}{4} H^{2} \Delta x^{2}\right)-2 \ln \left(\frac{\sqrt{e}}{4} H^{2} \Delta x^{2}\right)\right]\right\}+O(D-4) \cdot(81)
\end{array}
$$

We can now sum (70), (78) and (81) to obtain the final result for this sub-section,

$$
\begin{aligned}
-i M_{\delta A}^{2}\left(x ; x^{\prime}\right) & =\frac{i e^{2} H^{2} \mu^{D-4}}{2^{4} \pi^{\frac{D}{2}}}\left\{-\frac{2 \Gamma\left(\frac{D}{2}+1\right)}{(D-3)(D-4)}-4 \ln \left(\frac{H}{2 \mu}\right)+1\right\} a^{4} \delta^{D}\left(x-x^{\prime}\right) \\
& -\frac{e^{2} H^{4}}{2^{6} \pi^{4}}\left(a a^{\prime}\right)^{3}\left\{\partial_{0}^{2}\left[\ln ^{2}\left(2^{-2} H^{2} \Delta x^{2}\right)-\ln \left(2^{-2} H^{2} \Delta x^{2}\right)\right]\right. \\
& \left.+\frac{\partial^{2}}{2}\left[3 \ln ^{2}\left(2^{-2} H^{2} \Delta x^{2}\right)-5 \ln \left(2^{-2} H^{2} \Delta x^{2}\right)\right]\right\}+O(D-4) .
\end{aligned}
$$

Note that all the nonlocal terms proportional to $\left(a a^{\prime}\right)^{2}$ have combined to produce a finite, local term.

\subsubsection{Contributions from $i \delta \Delta_{B} \times i \Delta_{\mathrm{cf}}$}

In this sub-section we evaluate the contributions to the full 3-point diagram (43) by making the following replacements for the scalar and photon propagators,

$$
\begin{aligned}
i \Delta_{A}\left(x ; x^{\prime}\right) & \longrightarrow i \Delta_{\mathrm{cf}}\left(x ; x^{\prime}\right) \\
i\left[{ }_{\mu} \Delta_{\rho}\right]\left(x ; x^{\prime}\right) & \longrightarrow a a^{\prime} i \delta \Delta_{B}\left(x ; x^{\prime}\right) \bar{\eta}_{\mu \rho} .
\end{aligned}
$$

Here $\bar{\eta}_{\mu \rho}$ is the purely spatial part (25) of the Lorentz metric and $i \delta \Delta_{B}\left(x ; x^{\prime}\right)$ is the residual of the $B$-type propagator (36) after the conformal contribution has been subtracted,

$$
\begin{aligned}
i \delta \Delta_{B}\left(x ; x^{\prime}\right)= & \frac{H^{2} \Gamma\left(\frac{D}{2}\right)}{16 \pi^{\frac{D}{2}}} \frac{\left(a a^{\prime}\right)^{2-\frac{D}{2}}}{\Delta x^{D-4}}-\frac{H^{D-2}}{(4 \pi)^{\frac{D}{2}}} \frac{\Gamma(D-2)}{\Gamma\left(\frac{D}{2}\right)} \\
& +\frac{H^{D-2}}{(4 \pi)^{\frac{D}{2}}} \sum_{n=1}^{\infty}\left\{\frac{\Gamma\left(n+\frac{D}{2}\right)}{\Gamma(n+2)}\left(\frac{y}{4}\right)^{n-\frac{D}{2}+2}-\frac{\Gamma(n+D-2)}{\Gamma\left(n+\frac{D}{2}\right)}\left(\frac{y}{4}\right)^{n}\right\} .
\end{aligned}
$$

As was the case for the $i \delta \Delta_{A}\left(x ; x^{\prime}\right)$ contributions considered in the previous sub-section, this diagram is not sufficiently singular for the infinite series 
terms from $i \delta \Delta_{B}\left(x ; x^{\prime}\right)$ to make a nonzero contribution in the $D=4$ limit. Unlike $i \delta \Delta_{A}\left(x ; x^{\prime}\right)$, even the $n=0$ terms of $i \delta \Delta_{B}\left(x ; x^{\prime}\right)$ vanish for $D=4$. This means they can only contribute when multiplied by a divergence.

Making replacements (83) and (84) in the 3-point diagram (43) gives,

$$
\begin{aligned}
-i M_{\delta B}^{2}\left(x ; x^{\prime}\right)=-e^{2}\left(a a^{\prime}\right)^{D-1} & \left\{i \delta \Delta_{B} \partial_{i} \partial_{i}^{\prime} i \Delta_{\mathrm{cf}}+\partial_{i}\left[i \delta \Delta_{B} \partial_{i}^{\prime} i \delta \Delta_{\mathrm{cf}}\right]\right. \\
& \left.+\partial_{i}^{\prime}\left[i \delta \Delta_{B} \partial_{i} i \Delta_{\mathrm{cf}}\right]+\partial_{i} \partial_{i}^{\prime}\left[i \delta \Delta_{B} i \Delta_{\mathrm{cf}}\right]\right\} .
\end{aligned}
$$

Because all the derivatives are spatial we can extract the scale factors from $i \Delta_{\text {cf }}\left(x ; x^{\prime}\right)$. We can also convert $\partial_{i}^{\prime}$ to $-\partial_{i}$ and combine two terms to obtain,

$$
\begin{aligned}
-i M_{\delta B}^{2}( & \left.x ; x^{\prime}\right)=\frac{e^{2} \Gamma\left(\frac{D}{2}-1\right)}{4 \pi^{\frac{D}{2}}}\left(a a^{\prime}\right)^{\frac{D}{2}} \\
& \times\left\{i \delta \Delta_{B} \nabla^{2}\left(\frac{1}{\Delta x^{D-2}}\right)+2 \partial_{i}\left[i \delta \Delta_{B} \partial_{i}\left(\frac{1}{\Delta x^{D-2}}\right)\right]+\nabla^{2}\left(\frac{i \delta \Delta_{B}}{\Delta x^{D-2}}\right)\right\} .
\end{aligned}
$$

In analogy with the analysis of the previous sub-section we can label the three terms of this expression " 1 ", " $2+3$ " and " 4 ".

The only nonzero contribution comes from the first term of (87). To get it one merely substitutes the first line of (85) and then exploits some simple derivative identities,

$$
\begin{gathered}
-i M_{\delta B_{1}}^{2}\left(x ; x^{\prime}\right)=\frac{e^{2} H^{2}}{2^{6} \pi^{D}} \Gamma\left(\frac{D}{2}-1\right) \Gamma\left(\frac{D}{2}\right) \frac{\left(a a^{\prime}\right)^{2}}{\Delta x^{D-4}} \nabla^{2}\left(\frac{1}{\Delta x^{D-2}}\right) \\
-\frac{e^{2} H^{D-2}}{2^{D+2} \pi^{D}} \frac{\Gamma(D-2)}{\frac{D}{2}-1}\left(a a^{\prime}\right)^{\frac{D}{2}} \nabla^{2}\left(\frac{1}{\Delta x^{D-2}}\right)+O(D-4), \\
=\frac{e^{2} H^{2}}{2^{6} \pi^{D}} \frac{\Gamma\left(\frac{D}{2}-1\right) \Gamma\left(\frac{D}{2}\right)}{4(D-3)}\left(a a^{\prime}\right)^{2}\left[D \nabla^{2}-(D-1) \partial^{2}\right]\left(\frac{1}{\Delta x^{2 D-6}}\right) \\
-\frac{e^{2} H^{D-2}}{2^{D+2} \pi^{D}} \frac{\Gamma(D-2)}{\frac{D}{2}-1}\left(a a^{\prime}\right)^{\frac{D}{2}} \nabla^{2}\left(\frac{1}{\Delta x^{D-2}}\right)+O(D-4) .
\end{gathered}
$$

The last expression is completely integrable so we can take the limit $D=4$, at which stage the spatial derivative terms cancel and the d'Alembertian term gives a delta function,

$$
-i M_{\delta B_{1}}^{2}\left(x ; x^{\prime}\right)=-\frac{i 3 e^{2} H^{2}}{2^{6} \pi^{2}} a^{4} \delta^{4}\left(x-x^{\prime}\right)+O(D-4) .
$$


The middle term of (87) is integrable with $\partial_{i}$ extracted, so we can take $D=4$ immediately. This gives zero because $i \delta \Delta_{B}$ vanishes in $D=4$,

$$
-i M_{\delta B_{2+3}}^{2}\left(x ; x^{\prime}\right) \equiv \frac{e^{2} \Gamma\left(\frac{D}{2}-1\right)}{4 \pi^{\frac{D}{2}}}\left(a a^{\prime}\right)^{\frac{D}{2}} 2 \partial_{i}\left[i \delta \Delta_{B} \partial_{i}\left(\frac{1}{\Delta x^{D-2}}\right)\right]=O(D-4) .
$$

The same analysis and the same result pertains for the final term of (87),

$$
-i M_{\delta B_{4}}^{2}\left(x ; x^{\prime}\right) \equiv \frac{e^{2} \Gamma\left(\frac{D}{2}-1\right)}{4 \pi^{\frac{D}{2}}}\left(a a^{\prime}\right)^{\frac{D}{2}} \nabla^{2}\left(\frac{i \delta \Delta_{B}}{\Delta x^{D-2}}\right)=O(D-4) .
$$

Our final result for this sub-section is therefore,

$$
-i M_{\delta B}^{2}\left(x ; x^{\prime}\right)=-\frac{i 3 e^{2} H^{2}}{2^{6} \pi^{2}} a^{4} \delta^{4}\left(x-x^{\prime}\right)+O(D-4) .
$$

\subsubsection{Contributions from $i \delta \Delta_{C} \times i \Delta_{\mathrm{cf}}$}

In this sub-section we evaluate the contributions to the full 3-point diagram (43) by making the following replacements for the scalar and photon propagators,

$$
\begin{aligned}
i \Delta_{A}\left(x ; x^{\prime}\right) & \longrightarrow i \Delta_{\mathrm{cf}}\left(x ; x^{\prime}\right), \\
i\left[{ }_{\mu} \Delta_{\rho}\right]\left(x ; x^{\prime}\right) & \longrightarrow-a a^{\prime} i \delta \Delta_{C}\left(x ; x^{\prime}\right) \delta_{\mu}^{0} \delta_{\rho}^{0} .
\end{aligned}
$$

The result is,

$$
\begin{array}{r}
-i M_{\delta C}^{2}\left(x ; x^{\prime}\right)=e^{2}\left(a a^{\prime}\right)^{D-1} i \delta \Delta_{C} \partial_{0} \partial_{0}^{\prime} i \Delta_{\mathrm{cf}}+e^{2} a^{\prime D-1} \partial_{0}\left[a^{D-1} i \delta \Delta_{C} \partial_{0}^{\prime} i \Delta_{\mathrm{cf}}\right] \\
+e^{2} a^{D-1} \partial_{0}^{\prime}\left[a^{\prime D-1} i \delta \Delta_{C} \partial_{0} i \Delta_{\mathrm{cf}}\right]+e^{2} \partial_{0} \partial_{0}^{\prime}\left[\left(a a^{\prime}\right)^{D-1} i \delta \Delta_{C} i \Delta_{\mathrm{cf}}\right] .
\end{array}
$$

Here $i \delta \Delta_{C}\left(x ; x^{\prime}\right)$ is the residual of the $C$-type propagator (37) after the conformal contribution has been subtracted,

$$
\begin{aligned}
& i \delta \Delta_{C}\left(x ; x^{\prime}\right)=\frac{H^{2}}{16 \pi^{\frac{D}{2}}}\left(\frac{D}{2}-3\right) \Gamma\left(\frac{D}{2}-1\right) \frac{\left(a a^{\prime}\right)^{2-\frac{D}{2}}}{\Delta x^{D-4}}+\frac{H^{D-2}}{(4 \pi)^{\frac{D}{2}}} \frac{\Gamma(D-3)}{\Gamma\left(\frac{D}{2}\right)} \\
& -\frac{H^{D-2}}{(4 \pi)^{\frac{D}{2}}} \sum_{n=1}^{\infty}\left\{\left(n-\frac{D}{2}+3\right) \frac{\Gamma\left(n+\frac{D}{2}-1\right)}{\Gamma(n+2)}\left(\frac{y}{4}\right)^{n-\frac{D}{2}+2}-(n+1) \frac{\Gamma(n+D-3)}{\Gamma\left(n+\frac{D}{2}\right)}\left(\frac{y}{4}\right)^{n}\right\} .
\end{aligned}
$$

As with the contributions from $i \delta \Delta_{B}\left(x ; x^{\prime}\right)$ considered in the previous subsection, the only way $i \delta \Delta_{C}\left(x ; x^{\prime}\right)$ can give a nonzero contribution in $D=4$ 
dimensions is for it to multiply a singular term. For (96) that means only the $n=0$ term can possibly contribute. Even for the $n=0$ term, both derivatives must act upon a $\Delta x^{2}$. If even a single derivative acts instead upon a scale factor, the result is a term which is integrable in $D=4$ dimensions, at which point the cancellations between pairs of terms evident in (97) results in zero net contribution. We can therefore extract the scale factors from $i \Delta_{\mathrm{cf}}\left(x ; x^{\prime}\right)$ and replace $\partial_{0}^{\prime}$ by $-\partial_{0}$ as we did in the previous sub-section,

$$
\begin{aligned}
-i M_{\delta C}^{2}\left(x ; x^{\prime}\right)= & -\frac{e^{2} \Gamma\left(\frac{D}{2}-1\right)}{4 \pi^{\frac{D}{2}}}\left(a a^{\prime}\right)^{\frac{D}{2}}\left\{i \delta \Delta_{C} \partial_{0}^{2}\left(\frac{1}{\Delta x^{D-2}}\right)\right. \\
& \left.+2 \partial_{0}\left[i \delta \Delta_{C} \partial_{0}\left(\frac{1}{\Delta x^{D-2}}\right)\right]+\partial_{0}^{2}\left(\frac{i \delta \Delta_{C}}{\Delta x^{D-2}}\right)\right\}+O(D-4) .
\end{aligned}
$$

The first term in (96) is reduced the same way as was the analogous first term from $i \delta \Delta_{B}\left(x ; x^{\prime}\right)$ in the previous sub-section,

$$
\begin{aligned}
& -i M_{\delta C_{1}}^{2}\left(x ; x^{\prime}\right) \equiv-\frac{e^{2} \Gamma\left(\frac{D}{2}-1\right)}{4 \pi^{\frac{D}{2}}}\left(a a^{\prime}\right)^{\frac{D}{2}} i \delta \Delta_{C} \partial_{0}^{2}\left(\frac{1}{\Delta x^{D-2}}\right) \\
& =-\frac{e^{2} H^{2}}{2^{6} \pi^{D}}\left(\frac{D}{2}-3\right) \Gamma^{2}\left(\frac{D}{2}-1\right) \frac{\left(a a^{\prime}\right)^{2}}{\Delta x^{D-4}} \partial_{0}^{2}\left(\frac{1}{\Delta x^{D-2}}\right) \\
& \quad-\frac{e^{2} H^{D-2}}{2^{D+2} \pi^{D}} \frac{\Gamma(D-3)}{\frac{D}{2}-1}\left(a a^{\prime}\right)^{\frac{D}{2}} \partial_{0}^{2}\left(\frac{1}{\Delta x^{D-2}}\right)+O(D-4), \\
& =-\frac{e^{2} H^{2}}{2^{6} \pi^{D}} \frac{\left(\frac{D}{2}-3\right) \Gamma^{2}\left(\frac{D}{2}-1\right)}{4(D-3)}\left(a a^{\prime}\right)^{2}\left[D \partial_{0}^{2}+\partial^{2}\right]\left(\frac{1}{\Delta x^{2 D-6}}\right) \\
& \quad-\frac{e^{2} H^{D-2}}{2^{D+2} \pi^{D}} \frac{\Gamma(D-3)}{\frac{D}{2}-1}\left(a a^{\prime}\right)^{\frac{D}{2}} \partial_{0}^{2}\left(\frac{1}{\Delta x^{D-2}}\right)+O(D-4), \\
& =\frac{e^{2} H^{2}}{2^{8} \pi^{4}}\left(a a^{\prime}\right)^{2} \partial^{2}\left(\frac{1}{\Delta x^{2}}\right)+O(D-4) \\
& =\frac{i e^{2} H^{2}}{2^{6} \pi^{2}} a^{4} \delta^{4}\left(x-x^{\prime}\right)+O(D-4) .
\end{aligned}
$$

As in the previous sub-section, the other terms are zero because they start out with enough derivatives extracted that we can take $D=4$ right away,

$$
-i M_{\delta C_{2+3}}^{2}\left(x ; x^{\prime}\right) \equiv-\frac{e^{2} \Gamma\left(\frac{D}{2}-1\right)}{4 \pi^{\frac{D}{2}}}\left(a a^{\prime}\right)^{\frac{D}{2}} 2 \partial_{0}\left[i \delta \Delta_{C} \partial_{0}\left(\frac{1}{\Delta x^{D-2}}\right)\right]=O(D-4)
$$




$$
-i M_{\delta C_{4}}^{2}\left(x ; x^{\prime}\right) \equiv-\frac{e^{2} \Gamma\left(\frac{D}{2}-1\right)}{4 \pi^{\frac{D}{2}}}\left(a a^{\prime}\right)^{\frac{D}{2}} \partial_{0}^{2}\left(\frac{i \delta \Delta_{C}}{\Delta x^{D-2}}\right)=O(D-4) .
$$

The final answer for this sub-section is therefore,

$$
-i M_{\delta C}^{2}\left(x ; x^{\prime}\right)=\frac{i e^{2} H^{2}}{2^{6} \pi^{2}} a^{4} \delta^{4}\left(x-x^{\prime}\right)+O(D-4) .
$$

\subsection{Renormalization}

As we have explained, no other terms can give nonzero contributions for $D=4$. The total for $-i M^{2}\left(x ; x^{\prime}\right)$ at one loop order is therefore the sum of (42), (62), (82), (93) and (106),

$$
\begin{aligned}
& -i M^{2}\left(x ; x^{\prime}\right)= \\
& \frac{i e^{2} \mu^{D-4}}{4 \pi^{\frac{D}{2}}} \frac{\Gamma\left(\frac{D}{2}-1\right)}{(D-3)(D-4)} a a^{\prime} \partial^{2} \delta^{D}\left(x-x^{\prime}\right)-\frac{i e^{2} \mu^{D-4}}{8 \pi^{\frac{D}{2}}}\left\{\frac{\Gamma\left(\frac{D}{2}+1\right)}{(D-3)(D-4)}\right. \\
& \left.+2 \ln \left(\frac{H}{2 \mu}\right)-\frac{1}{2}\right\} H^{2} a^{4} \delta^{D}\left(x-x^{\prime}\right)-\frac{e^{2}}{2^{8} \pi^{4}} a a^{\prime} \partial^{6}\left\{\ln ^{2}\left(\mu^{2} \Delta x^{2}\right)-2 \ln \left(\mu^{2} \Delta x^{2}\right)\right\} \\
& -\frac{e^{2} H^{4}}{2^{6} \pi^{4}}\left(a a^{\prime}\right)^{3}\left\{\partial_{0}^{2}\left[\ln ^{2}\left(2^{-2} H^{2} \Delta x^{2}\right)-\ln \left(2^{-2} H^{2} \Delta x^{2}\right)\right]\right. \\
& \left.+\frac{\partial^{2}}{2}\left[3 \ln ^{2}\left(2^{-2} H^{2} \Delta x^{2}\right)-5 \ln \left(2^{-2} H^{2} \Delta x^{2}\right)\right]\right\}+O(D-4) \cdot(107)
\end{aligned}
$$

By simply deleting (82) we can also obtain the one loop self-mass-squared for a conformally coupled scalar,

$$
\begin{aligned}
-i \mathcal{M}^{2}\left(x ; x^{\prime}\right)= & \frac{i e^{2} \mu^{D-4}}{4 \pi^{\frac{D}{2}}} \frac{\Gamma\left(\frac{D}{2}-1\right)}{(D-3)(D-4)} a a^{\prime} \partial^{2} \delta^{D}\left(x-x^{\prime}\right) \\
& -\frac{e^{2}}{2^{8} \pi^{4}} a a^{\prime} \partial^{6}\left\{\ln ^{2}\left(\mu^{2} \Delta x^{2}\right)-2 \ln \left(\mu^{2} \Delta x^{2}\right)\right\}+O(D-4) .(10
\end{aligned}
$$

The relevant counterterms (91) give rise to a diagram with the topology depicted in Fig. 3. The contribution it makes is,

$$
\begin{array}{r}
-i M_{\mathrm{ctm}}^{2}\left(x ; x^{\prime}\right)=i \delta Z_{2} \partial_{\mu}\left(\sqrt{-g} g^{\mu \nu} \partial_{\nu}\right) \delta^{D}\left(x-x^{\prime}\right)-i \delta \xi R \sqrt{-g} \delta^{D}\left(x-x^{\prime}\right) \\
=i \delta Z_{2}\left(a a^{\prime}\right)^{\frac{D}{2}-1} \partial^{2} \delta^{D}\left(x-x^{\prime}\right) \\
-i\left[\delta \xi-\frac{1}{4}\left(\frac{D-2}{D-1}\right) \delta Z_{2}\right](D-1) D H^{2} a^{D} \delta^{D}\left(x-x^{\prime}\right)
\end{array}
$$




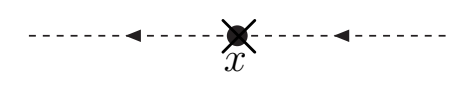

Fig. 3: Contribution from counterterms.

By comparing (107) with (110) we see that the simplest choice of counterterms is,

$$
\begin{aligned}
\delta Z_{2} & =-\frac{e^{2} \mu^{D-4}}{4 \pi^{\frac{D}{2}}} \frac{\Gamma\left(\frac{D}{2}-1\right)}{(D-3)(D-4)}, i \\
\delta \xi & =-\frac{e^{2} \mu^{D-4}}{8 \pi^{\frac{D}{2}}(D-1) D}\left\{\frac{3 \Gamma\left(\frac{D}{2}+1\right)}{(D-3)(D-4)}+2 \ln \left(\frac{H}{2 \mu}\right)-\frac{1}{2}\right\} .
\end{aligned}
$$

This gives the following fully renormalized result,

$$
\begin{aligned}
& -i M_{\mathrm{ren}}^{2}\left(x ; x^{\prime}\right)=-\frac{i e^{2}}{8 \pi^{2}} a a^{\prime} \ln \left(a a^{\prime}\right) \partial^{2} \delta^{4}\left(x-x^{\prime}\right)+\frac{i e^{2} H^{2}}{4 \pi^{2}} a^{4} \ln (a) \delta^{4}\left(x-x^{\prime}\right) \\
& -\frac{e^{2}}{2^{8} \pi^{4}} a a^{\prime} \partial^{6}\left\{\ln ^{2}\left(\mu^{2} \Delta x^{2}\right)-2 \ln \left(\mu^{2} \Delta x^{2}\right)\right\}-\frac{e^{2} H^{4}}{2^{6} \pi^{4}}\left(a a^{\prime}\right)^{3}\left\{\partial _ { 0 } ^ { 2 } \left[\ln ^{2}\left(2^{-2} H^{2} \Delta x^{2}\right)\right.\right. \\
& \left.\left.\quad-\ln \left(2^{-2} H^{2} \Delta x^{2}\right)\right]+\frac{\partial^{2}}{2}\left[3 \ln ^{2}\left(2^{-2} H^{2} \Delta x^{2}\right)-5 \ln \left(2^{-2} H^{2} \Delta x^{2}\right)\right]\right\} .(113)
\end{aligned}
$$

To renormalize the self-mass-squared of a conformally coupled scalar (108) the best choice of counterterms would be,

$$
\left.\delta Z_{2}\right|_{\mathrm{conf}}=\left.4\left(\frac{D-1}{D-2}\right) \delta \xi\right|_{\mathrm{conf}}=-\frac{e^{2} \mu^{D-4}}{4 \pi^{\frac{D}{2}}} \frac{\Gamma\left(\frac{D}{2}-1\right)}{(D-3)(D-4)} .
$$

With this choice we get the following renormalized self-mass-squared,

$$
\begin{aligned}
-i \mathcal{M}_{\mathrm{ren}}^{2}\left(x ; x^{\prime}\right)=-\frac{i e^{2}}{8 \pi^{2}} a a^{\prime} & \ln \left(a a^{\prime}\right) \partial^{2} \delta^{4}\left(x-x^{\prime}\right) \\
& -\frac{e^{2}}{2^{8} \pi^{4}} a a^{\prime} \partial^{6}\left\{\ln ^{2}\left(\mu^{2} \Delta x^{2}\right)-2 \ln \left(\mu^{2} \Delta x^{2}\right)\right\} .
\end{aligned}
$$

The vastly greater complexity of the minimally coupled result (113) derives from inflationary particle production, which the conformally coupled scalar does not experience. It is also worth noting that the conformally coupled 
result can be put in a manifestly de Sitter invariant form using the de Sitter length function $y\left(x ; x^{\prime}\right)$ (29) and the conformal d'Alembertian,

$$
\mathcal{D}_{\mathrm{cf}} \equiv \partial_{\mu}\left(\sqrt{-g} g^{\mu \nu} \partial_{\nu}\right)-\frac{1}{6} R \sqrt{-g}=a \partial^{2} a
$$

The resulting expression is,

$$
-i \mathcal{M}_{\text {ren }}^{2}\left(x ; x^{\prime}\right)=-\frac{e^{2} H^{2}}{32 \pi^{4}} \mathcal{D}_{\mathrm{cf}} \mathcal{D}_{\mathrm{cf}}^{\prime}\left\{\frac{\ln \left[y\left(x ; x^{\prime}\right) \mu^{2} / H^{2}\right]}{y\left(x ; x^{\prime}\right)}\right\} .
$$

Note its similarity to one loop scalar self-mass-squared found for a Yukawacoupled scalar [15]. The manifest de Sitter invariance of this expression proves that our use of a noninvariant gauge poses no problem at least at one loop order.

\section{$4 \quad M^{2}\left(x ; x^{\prime}\right)$ in Allen-Jacobson Gauge}

One can see from expression (14) for the electromagnetic Lagrangian that a possible invariant gauge fixing term is,

$$
\mathcal{L}_{\mathrm{AJ}}=-\frac{1}{2}\left(g^{\mu \nu} A_{\mu ; \nu}\right)^{2} \sqrt{-g}=-\frac{1}{2} a^{D-4}\left(\eta^{\mu \nu} A_{\mu, \nu}-(D-2) H a A_{0}\right)^{2} .
$$

In this gauge the photon propagator obeys,

$$
\sqrt{-g}\left[\left(D^{2}\right)_{\rho}^{\mu}-R_{\rho}^{\mu}\right] i\left[{ }^{\rho} \Delta_{\nu}\right]\left(x ; x^{\prime}\right)=\delta_{\nu}^{\mu} i \delta^{D}\left(x-x^{\prime}\right),
$$

where the contravariant vector covariant derivative operator is,

$$
\begin{aligned}
\left(D^{2}\right)_{\rho}^{\mu} \equiv g^{\alpha \beta}\left\{\delta_{\rho}^{\mu} \partial_{\alpha} \partial_{\beta}+\Gamma_{\alpha \rho}^{\mu} \partial_{\beta}+\Gamma_{\beta \rho}^{\mu} \partial_{\alpha}\right. & \\
& \left.-\delta_{\rho}^{\mu} \Gamma_{\alpha \beta}^{\nu} \partial_{\nu}+\Gamma_{\alpha \rho, \beta}^{\mu}+\Gamma_{\alpha \nu}^{\mu} \Gamma_{\beta \rho}^{\nu}-\Gamma_{\nu \rho}^{\mu} \Gamma_{\alpha \beta}^{\nu}\right\} .
\end{aligned}
$$

Of course equation (119) is generally covariant in addition to being de Sitter invariant, but we will only solve it for the special case of de Sitter background.

Allen and Jacobson expressed their result for the solution of (119) in terms of scalar functions multiplying, respectively, the parallel transport matrix and the product of two gradients of $\ell\left(x ; x^{\prime}\right)$ [18]. For our purposes it is more 
effective to express the same result in terms of the length function $y\left(x ; x^{\prime}\right)$ — which was defined in (29) - and its derivatives, ${ }^{1}$

$$
i\left[{ }_{\mu} \Delta_{\nu}^{\mathrm{AJ}}\right]\left(x ; x^{\prime}\right)=B(y) \frac{\partial^{2} y}{\partial x^{\mu} \partial x^{\prime \nu}}+C(y) \frac{\partial y}{\partial x^{\mu}} \frac{\partial y}{\partial x^{\prime \nu}},
$$

It is straightforward to compute various derivatives of $y\left(x ; x^{\prime}\right)$ in conformal coordinates,

$$
\begin{aligned}
\frac{\partial y}{\partial x^{\mu}} & =H a\left(y \delta_{\mu}^{0}+2 a^{\prime} H \Delta x_{\mu}\right) \\
\frac{\partial y}{\partial x^{\prime \nu}} & =H a^{\prime}\left(y \delta_{\nu}^{0}-2 a H \Delta x_{\nu}\right) \\
\frac{\partial^{2} y}{\partial x^{\mu} \partial x^{\prime \nu}} & =H^{2} a a^{\prime}\left(y \delta_{\mu}^{0} \delta_{\nu}^{0}-2 \delta_{\mu}^{0} a H \Delta x_{\nu}+2 a^{\prime} H \Delta x_{\mu} \delta_{\nu}^{0}-2 \eta_{\mu \nu}\right),
\end{aligned}
$$

where the contravariant interval is $\Delta x_{\mu} \equiv \eta_{\mu \nu}\left(x^{\nu}-x^{\prime \nu}\right)$. One can also establish the following useful results in any coordinate system,

$$
\begin{aligned}
g^{\mu \nu}(x) \frac{\partial y}{\partial x^{\mu}} \frac{\partial y}{\partial x^{\nu}} & =H^{2}\left(4 y-y^{2}\right)=g^{\mu \nu}\left(x^{\prime}\right) \frac{\partial y}{\partial x^{\prime \mu}} \frac{\partial y}{\partial x^{\prime \nu}}, \\
g^{\mu \nu}(x) \frac{\partial y}{\partial x^{\nu}} \frac{\partial^{2} y}{\partial x^{\mu} \partial x^{\prime \sigma}} & =H^{2}(2-y) \frac{\partial y}{\partial x^{\prime \sigma}}, \\
g^{\rho \sigma}\left(x^{\prime}\right) \frac{\partial y}{\partial x^{\prime \sigma}} \frac{\partial^{2} y}{\partial x^{\mu} \partial x^{\prime \rho}} & =H^{2}(2-y) \frac{\partial y}{\partial x^{\mu}} \\
g^{\mu \nu}(x) \frac{\partial^{2} y}{\partial x^{\mu} \partial x^{\prime \rho}} \frac{\partial^{2} y}{\partial x^{\nu} \partial x^{\prime \sigma}} & =4 H^{4} g_{\rho \sigma}\left(x^{\prime}\right)-H^{2} \frac{\partial y}{\partial x^{\prime \rho}} \frac{\partial y}{\partial x^{\prime \sigma}}
\end{aligned}
$$

${ }^{1}$ One can show [16] that the parallel transport matrix and the product of the two gradients take the following form in terms of $y\left(x ; x^{\prime}\right)$ and its derivatives,

$$
\begin{aligned}
{\left[{ }_{\mu} g_{\nu}\right]\left(x ; x^{\prime}\right) } & =-\frac{1}{2 H^{2}} \frac{\partial^{2} y}{\partial x^{\mu} \partial x^{\prime \nu}}-\frac{1}{2 H^{2}(4-y)} \frac{\partial y}{\partial x^{\mu}} \frac{\partial y}{\partial x^{\prime \nu}}, \\
{\left[{ }_{\mu} n\right]\left(x ; x^{\prime}\right)\left[n_{\nu}\right]\left(x ; x^{\prime}\right) } & =\frac{1}{H^{2} y(4-y)} \frac{\partial y}{\partial x^{\mu}} \frac{\partial y}{\partial x^{\prime \nu}} .
\end{aligned}
$$

Hence the functions $\alpha$ and $\beta$ of Allen and Jacobson [18 relate to our functions $B(y)$ and $C(y)$ as follows,

$$
\begin{aligned}
\alpha & =-2 H^{2} B(y) \\
\beta & =-y H^{2} B(y)+y(4-y) H^{2} C(y) .
\end{aligned}
$$




$$
\begin{aligned}
g^{\rho \sigma}\left(x^{\prime}\right) \frac{\partial^{2} y}{\partial x^{\mu} \partial x^{\prime \rho}} \frac{\partial^{2} y}{\partial x^{\nu} \partial x^{\prime \sigma}} & =4 H^{4} g_{\mu \nu}(x)-H^{2} \frac{\partial y}{\partial x^{\mu}} \frac{\partial y}{\partial x^{\nu}}, \\
\frac{\partial^{2} y}{D x^{\mu} D x^{\nu}} & =H^{2}(2-y) g_{\mu \nu}(x), \\
\frac{\partial^{3} y}{D x^{\mu} D x^{\nu} \partial x^{\prime \rho}} & =-H^{2} g_{\mu \nu}(x) \frac{\partial y}{\partial x^{\prime \rho}}, \\
D^{2} \frac{\partial^{2} y}{\partial x^{\mu} \partial x^{\prime \nu}} & =-H^{2} \frac{\partial^{2} y}{\partial x^{\mu} \partial x^{\prime \nu}} .
\end{aligned}
$$

Substituting (121) into (119) and making use of the various identities gives the following coupled, ordinary differential equations for $B(y)$ and $C(y)$,

$$
\begin{aligned}
\left(4 y-y^{2}\right) B^{\prime \prime}+D(2-y) B^{\prime}-D B+(4-2 y) C & =0 \\
\left(4 y-y^{2}\right) C^{\prime \prime}+(D+4)(2-y) C^{\prime}-2 D C-2 B^{\prime} & =0
\end{aligned}
$$

This coupling is one indication of the substantially greater complication of working with a de Sitter invariant gauge. We solve this system the same way Allen and Jacobson solved their analogous system [18, by partially decoupling through the change of variables $F(y) \equiv B^{\prime}(y)-C(y)$,

$$
\begin{aligned}
& \left(4 y-y^{2}\right) F^{\prime \prime}+(D+2)(2-y) F^{\prime}-2(D-1) F=0, \\
& \left(4 y-y^{2}\right) B^{\prime \prime}+(D+2)(2-y) B^{\prime}-D B=(4-2 y) F .
\end{aligned}
$$

One then substitutes the general solution for $F(y)$ into the second equation. ${ }^{2}$ The various integration constants are chosen to enforce the delta function singularity at $y=0$ and analyticity at $y=4$. In the end one finds,

$$
\begin{aligned}
B(y)= & \frac{H^{D-4}}{(4 \pi)^{\frac{D}{2}}} \frac{\Gamma(D-3)}{\Gamma\left(\frac{D}{2}\right)}\left\{-\left(\frac{D-3}{2}\right){ }_{2} F_{1}\left(D-2,1 ; \frac{D}{2} ; 1-\frac{y}{4}\right)\right. \\
& +\left[\frac{1}{2}-\left(\frac{D-2}{D}\right)(\psi(D)-\psi(1))\right]{ }_{2} F_{1}\left(D, 1 ; \frac{D}{2}+1 ; \frac{y}{4}\right)
\end{aligned}
$$

\footnotetext{
${ }^{2}$ The two linearly independent solutions to the $F$ equation (135) are,
}

$$
{ }_{2} F_{1}\left(D-1,2 ; \frac{D}{2}+1 ; 1-\frac{y}{4}\right) \quad \text { and } \quad{ }_{2} F_{1}\left(D-1,2 ; \frac{D}{2}+1 ; \frac{y}{4}\right) .
$$

The two homogeneous equations to the $B$ equation (136) are,

$$
{ }_{2} F_{1}\left(D, 1 ; \frac{D}{2}+1 ; 1-\frac{y}{4}\right) \quad \text { and } \quad{ }_{2} F_{1}\left(D, 1 ; \frac{D}{2}+1 ; \frac{y}{4}\right) .
$$




$$
\begin{aligned}
& \left.+\frac{(D-3)}{\left(4 y-y^{2}\right)^{\frac{D}{2}}} \int_{0}^{y} d y^{\prime}\left(4 y^{\prime}-y^{\prime 2}\right)^{\frac{D}{2}-1} \int_{y^{\prime}}^{4} d y_{2}{ }_{2} F_{1}\left(D-2,1 ; \frac{D}{2} ; 1-\frac{y^{\prime \prime}}{4}\right)\right\},(137) \\
C(y)= & \frac{H^{D-4}}{(4 \pi)^{\frac{D}{2}}} \frac{\Gamma(D-3)}{\Gamma\left(\frac{D}{2}\right)} \frac{\partial}{\partial y}\left\{\left[\frac{1}{2}-\left(\frac{D-2}{D}\right)(\psi(D)-\psi(1))\right]_{2} F_{1}\left(D, 1 ; \frac{D}{2}+1 ; \frac{y}{4}\right)\right. \\
& \left.+\frac{(D-3)}{\left(4 y-y^{2}\right)^{\frac{D}{2}}} \int_{0}^{y} d y^{\prime}\left(4 y^{\prime}-y^{\prime 2}\right)^{\frac{D}{2}-1} \int_{y^{\prime}}^{4} d y^{\prime \prime}{ }_{2} F_{1}\left(D-2,1 ; \frac{D}{2} ; 1-\frac{y^{\prime \prime}}{4}\right)\right\},(138) \\
F(y)= & \frac{H^{D-4}}{(4 \pi)^{\frac{D}{2}}} \frac{\Gamma(D-3)}{\Gamma\left(\frac{D}{2}\right)} \times-\left(\frac{D-3}{2}\right) \frac{\partial}{\partial y}{ }_{2} F_{1}\left(D-2,1 ; \frac{D}{2} ; 1-\frac{y}{4}\right), \\
= & -\frac{H^{D-4}}{(4 \pi)^{\frac{D}{2}}} \frac{\Gamma(D-1)}{\Gamma\left(\frac{D}{2}\right)} \frac{\partial}{\partial y}\left\{\frac{1}{\left(4 y-y^{2}\right)^{\frac{D}{2}-1}} \int_{y}^{4} d y^{\prime}\left(4 y^{\prime}-y^{\prime 2}\right)^{\frac{D}{2}-2}\right\},
\end{aligned}
$$

where $\psi(z) \equiv \Gamma^{\prime}(z) / \Gamma(z)$.

Simplicity is sometimes a matter of taste but it is difficult to imagine any criterion by which the propagator in this gauge is simpler than the one we used in the previous section. Where the de Sitter invariant formalism has a clear advantage is in taking derivatives and contracting indicies. This is because derivatives of a function of $y\left(x ; x^{\prime}\right)$, such as $B(y)$ or $C(y)$, produce ordinary derivatives of these functions with respect to $y$ times the same basis tensors as in the photon propagator. Invariant contractions of these basis tensors always reduce to functions or $y$. For example, one can easily compute the divergence of the photon propagator on $x^{\nu}$,

$$
\begin{aligned}
\partial_{\nu}\left(\sqrt{-g(x)} g^{\mu \nu}(x)\right. & \left.i\left[{ }_{\mu} \Delta_{\rho}^{\mathrm{AJ}}\right]\left(x ; x^{\prime}\right)\right)=H^{2} a^{D} \frac{\partial y}{\partial x^{\prime \rho}} \\
& \times\left\{(2-y) B^{\prime}-D B+\left(4 y-y^{2}\right) C^{\prime}+(D+1)(2-y) C\right\} .
\end{aligned}
$$

By first substituting $C(y)=B^{\prime}(y)-F(y)$ and then using the $B$ equation (136) we can actually express this in terms of $F(y)$ and its first derivative,

$$
\begin{aligned}
& (2-y) B^{\prime}-D B+\left(4 y-y^{2}\right) C^{\prime}+(D+1)(2-y) C \\
& =\left(4 y-y^{2}\right) B^{\prime \prime}+(D+2)(2-y) B^{\prime}-D B-\left(4 y-y^{2}\right) F^{\prime}-(D+1)(2-y) F, \\
& =-\left(4 y-y^{2}\right) F^{\prime}-(D-1)(2-y) F .
\end{aligned}
$$

Of course a similar result pertains for the divergence on $x^{\prime \sigma}$,

$$
\partial_{\sigma}^{\prime}\left(\sqrt{-g\left(x^{\prime}\right)} g^{\rho \sigma}\left(x^{\prime}\right) i\left[{ }_{\mu} \Delta_{\rho}^{\mathrm{AJ}}\right]\left(x ; x^{\prime}\right)\right)
$$




$$
=-H^{2} a^{\prime D} \frac{\partial y}{\partial x^{\mu}}\left\{\left(4 y-y^{2}\right) F^{\prime}+(D-1)(2-y) F\right\} .
$$

When both divergences are taken the result can be reduced to a scalar, then the second derivatives of $F(y)$ can be removed using the $F$ equation (135),

$$
\begin{gathered}
\partial_{\nu} \partial_{\sigma}^{\prime}\left(\sqrt{-g(x)} g^{\mu \nu}(x) \sqrt{-g\left(x^{\prime}\right)} g^{\rho \sigma}\left(x^{\prime}\right) i\left[{ }_{\mu} \Delta_{\rho}^{\mathrm{AJ}}\right]\left(x ; x^{\prime}\right)\right)=-a^{D} \delta^{D}\left(x-x^{\prime}\right) \\
-(D-1) H^{4}\left(a a^{\prime}\right)^{D}\left\{(2-y)\left(4 y-y^{2}\right) F^{\prime}+(D-1)(2-y)^{2} F+4 F\right\} .
\end{gathered}
$$

To take advantage of these relations we re-express the 3-point contribution (43) as follows,

$$
\begin{aligned}
-i M_{3 \mathrm{AJ}}^{2}\left(x ; x^{\prime}\right)= & \left.-4 e^{2} \sqrt{-g} g^{\mu \nu} \sqrt{-g^{\prime}} g^{\prime \rho \sigma} i{ }_{\mu} \Delta_{\rho}^{\mathrm{AJ}}\right]\left(x ; x^{\prime}\right) \partial_{\nu} \partial_{\sigma}^{\prime} i \Delta_{A}\left(x ; x^{\prime}\right) \\
& \left.-2 e^{2} \partial_{\nu}\left(\sqrt{-g} g^{\mu \nu} i{ }_{\mu} \Delta_{\rho}^{\mathrm{AJ}}\right]\left(x ; x^{\prime}\right)\right) \sqrt{-g^{\prime}} g^{\prime \rho \sigma} \partial_{\sigma}^{\prime} i \Delta_{A}\left(x ; x^{\prime}\right) \\
& \left.-2 e^{2} \partial_{\sigma}^{\prime}\left(\sqrt{-g^{\prime}} g^{\prime \rho \sigma} i{ }_{\mu} \Delta_{\rho}^{\mathrm{AJ}}\right]\left(x ; x^{\prime}\right)\right) \sqrt{-g} g^{\mu \nu} \partial_{\nu} i \Delta_{A}\left(x ; x^{\prime}\right) \\
& -e^{2} \partial_{\nu} \partial_{\sigma}^{\prime}\left(\sqrt{-g} g^{\mu \nu} \sqrt{-g^{\prime}} g^{\prime \rho \sigma} i\left[{ }_{\mu} \Delta_{\rho}^{\mathrm{AJ}}\right]\left(x ; x^{\prime}\right)\right) i \Delta_{A}\left(x ; x^{\prime}\right) .
\end{aligned}
$$

Because the scalar propagator is not quite de Sitter invariant we must also extract its de Sitter breaking term,

$$
i \Delta_{A}\left(x ; x^{\prime}\right) \equiv A(y)+k \ln \left(a a^{\prime}\right) \quad \text { where } \quad k \equiv \frac{H^{D-2}}{(4 \pi)^{\frac{D}{2}}} \frac{\Gamma(D-1)}{\Gamma\left(\frac{D}{2}\right)} .
$$

The necessary first and second derivatives are,

$$
\begin{aligned}
\partial_{\nu} i \Delta_{A}\left(x ; x^{\prime}\right) & =\frac{\partial y}{\partial x^{\nu}} A^{\prime}(y)+k H a \delta_{\nu}^{0}, \\
\partial_{\sigma}^{\prime} i \Delta_{A}\left(x ; x^{\prime}\right)= & \frac{\partial y}{\partial x^{\prime \sigma}} A^{\prime}(y)+k H a^{\prime} \delta_{\sigma}^{0}, \\
\partial_{\nu} \partial_{\sigma}^{\prime} i \Delta_{A}\left(x ; x^{\prime}\right)= & \frac{\partial^{2} y}{\partial x^{\nu} \partial x^{\prime \sigma}} A^{\prime}(y)+\frac{\partial y}{\partial x^{\nu}} \frac{\partial y}{\partial x^{\prime \sigma}} A^{\prime \prime}(y) \\
& +\delta_{\nu}^{0} \delta_{\sigma}^{0} a^{2-D} i \delta^{D}\left(x-x^{\prime}\right) .
\end{aligned}
$$


Note also that the function $A(y)$ obeys the inhomogeneous equation,

$$
\left(4 y-y^{2}\right) A^{\prime \prime}+D(2-y) A^{\prime}-(D-1) k=0 .
$$

With these identities, and some of the previous ones for the photon propagator and contractions, the first term of (146) reduces to,

$$
\begin{gathered}
\left.-i M_{3 \mathrm{AJ}_{1}}^{2}\left(x ; x^{\prime}\right) \equiv-4 e^{2} \sqrt{-g} g^{\mu \nu} \sqrt{-g^{\prime}} g^{\prime \rho \sigma} i{ }_{\mu} \Delta_{\rho}^{\mathrm{AJ}}\right]\left(x ; x^{\prime}\right) \partial_{\nu} \partial_{\sigma}^{\prime} i \Delta_{A}\left(x ; x^{\prime}\right), \\
=-i 8 e^{2} H^{2} B a^{D} \delta^{D}\left(x-x^{\prime}\right)-4 e^{2} H^{4}\left(a a^{\prime}\right)^{D}\left\{\left[4 D-\left(4 y-y^{2}\right)\right] A^{\prime} B\right. \\
\left.+(2-y)\left(4 y-y^{2}\right) A^{\prime} C+(2-y)\left(4 y-y^{2}\right) A^{\prime \prime} B+\left(4 y-y^{2}\right)^{2} A^{\prime \prime} C\right\},(15 \\
=-i 8 e^{2} H^{2} B a^{D} \delta^{D}\left(x-x^{\prime}\right)+4 k(D-1) e^{2} H^{4}\left(a a^{\prime}\right)^{D}\left[-(2-y) B-\left(4 y-y^{2}\right) C\right] \\
+4(D-1) e^{2} H^{4}\left(a a^{\prime}\right)^{D}\left(4 y-y^{2}\right) A^{\prime}[-B+(2-y) C] .(15
\end{gathered}
$$

The second term of (146) gives,

$$
\begin{aligned}
-i M_{3 \mathrm{AJ}_{2}}^{2}\left(x ; x^{\prime}\right) \equiv & -2 e^{2} \partial_{\nu}\left(\sqrt{-g} g^{\mu \nu} i\left[{ }_{\mu} \Delta_{\rho}^{\mathrm{AJ}}\right]\left(x ; x^{\prime}\right)\right) \sqrt{-g^{\prime}} g^{\prime \rho \sigma} \partial_{\sigma}^{\prime} i \Delta_{A}\left(x ; x^{\prime}\right),(15 \\
= & 2 e^{2} H^{4}\left(a a^{\prime}\right)^{D}\left\{\left(4 y-y^{2}\right) A^{\prime}+k\left[2-y-2 \frac{a}{a^{\prime}}\right]\right\} \\
& \times\left\{\left(4 y-y^{2}\right) F^{\prime}+(D-1)(2-y) F\right\} .(15
\end{aligned}
$$

The third term differs only by interchanging $x^{\mu}$ and $x^{\prime \mu}$, so the two sum to,

$$
\begin{aligned}
-i M_{3 \mathrm{AJ}_{2+3}}^{2}\left(x ; x^{\prime}\right)=4 e^{2} H^{4}\left(a a^{\prime}\right)^{D}\left\{\left(4 y-y^{2}\right) A^{\prime}-k\left[y+a a^{\prime} H^{2} \Delta \eta^{2}\right]\right\} \\
\times\left\{\left(4 y-y^{2}\right) F^{\prime}+(D-1)(2-y) F\right\} .
\end{aligned}
$$

The fourth term in (146) gives,

$$
\begin{array}{r}
-i M_{3 \mathrm{AJ}_{4}}^{2}\left(x ; x^{\prime}\right) \equiv-e^{2} \partial_{\nu} \partial_{\sigma}^{\prime}\left(\sqrt{-g} g^{\mu \nu} \sqrt{-g^{\prime}} g^{\prime \rho \sigma} i\left[_{\mu} \Delta_{\rho}^{\mathrm{AJ}}\right]\left(x ; x^{\prime}\right)\right) i \Delta_{A}\left(x ; x^{\prime}\right),( \\
=i e^{2}\{A+2 k \ln (a)\} a^{D} \delta^{D}\left(x-x^{\prime}\right)+(D-1) e^{2} H^{4}\left(a a^{\prime}\right)^{D}\left\{A+k \ln \left(a a^{\prime}\right)\right\} \\
\times\left\{(2-y)\left(4 y-y^{2}\right) F^{\prime}+(D-1)(2-y)^{2} F+4 F\right\} .(15
\end{array}
$$

Finally, we should include the 4-point contribution,

$$
\begin{aligned}
-i M_{4 \mathrm{AJ}}^{2}\left(x ; x^{\prime}\right) & \equiv-i e^{2} \sqrt{-g} g^{\mu \nu} i\left[{ }_{\mu} \Delta_{\nu}^{\mathrm{AJ}}\right]\left(x ; x^{\prime}\right) \delta^{D}\left(x-x^{\prime}\right), \\
& =i 2 D e^{2} H^{2} B a^{D} \delta^{D}\left(x-x^{\prime}\right) .
\end{aligned}
$$


Up to this point we have simply exploited the differential equations (151) and (135] [36) that any solution for the scalar and photon propagators must obey. Now we substitute the specific solutions for $A(y), B(y), C(y)$ and $F(y)$, and then expand the relevant combinations to include all singular and finite, nonzero contributions. The expansion of $A(y)$ can be read off from (31) and has the nice property of terminating for $D=4$. The two combinations we require are,

$$
\begin{aligned}
A(y) & =\frac{H^{D-2}}{4 \pi^{\frac{D}{2}}}\left\{\frac{\Gamma\left(\frac{D}{2}-1\right)}{y^{\frac{D}{2}-1}}-\frac{1}{2} \ln \left(\frac{y}{4}\right)-\frac{1}{4}+O(D-4)\right\}, \\
\left(4 y-y^{2}\right) A^{\prime}(y) & =-\frac{H^{D-2}}{4 \pi^{\frac{D}{2}}}\left\{\frac{4 \Gamma\left(\frac{D}{2}\right)}{y^{\frac{D}{2}-1}}+\frac{\left(\frac{D}{2}-1\right) \Gamma\left(\frac{D}{2}\right)}{y^{\frac{D}{2}-2}}-\frac{y}{2}+O(D-4)\right\} .
\end{aligned}
$$

The function $F(y)$ also terminates in $D=4$,

$$
\begin{array}{r}
F(y)=\frac{H^{D-4}}{16 \pi^{\frac{D}{2}}}\left\{\frac{2 \Gamma\left(\frac{D}{2}\right)}{y^{\frac{D}{2}}}+\frac{(D-4) \Gamma\left(\frac{D}{2}\right)}{2 y^{\frac{D}{2}-1}}+\frac{1}{2^{D-1}} \sum_{n=0}^{\infty}\left[(n+1) \frac{\Gamma(n+D-1)}{\Gamma\left(n+\frac{D}{2}+1\right)}\left(\frac{y}{4}\right)^{n}\right.\right. \\
\left.\left.-\left(n-\frac{D}{2}+3\right) \frac{\Gamma\left(n+\frac{D}{2}-2\right)}{(n+2) !}\left(\frac{y}{4}\right)^{n-\frac{D}{2}+2}\right]\right\} \cdot(164)
\end{array}
$$

Two combinations of it are needed,

$$
\begin{gathered}
\left(4 y-y^{2}\right) F^{\prime}+(D-1)(2-y) F=\frac{H^{D-4}}{16 \pi^{\frac{D}{2}}}\left\{-\frac{4 \Gamma\left(\frac{D}{2}\right)}{y^{\frac{D}{2}}}-\frac{\Gamma\left(\frac{D}{2}+1\right)}{y^{\frac{D}{2}-1}}+O(D-4)\right\}, \\
(2-y)\left(4 y-y^{2}\right) F^{\prime}+(D-1)(2-y)^{2} F+4 F=\frac{H^{D-4}}{16 \pi^{\frac{D}{2}}} \times \frac{\Gamma(D-1)}{2^{D-4} \Gamma\left(\frac{D}{2}\right)} .
\end{gathered}
$$

The last expression can be shown to be exact using (140).

Unlike $i \Delta_{B}\left(x ; x^{\prime}\right)$ and $i \Delta_{C}\left(x ; x^{\prime}\right)$, the expansions for $B(y)$ and $C(y)$ do not terminate in $D=4$ dimensions. (This is another indication of the complication associated with employing a de Sitter invariant gauge.) However, we can take $D=4$ in those terms which vanish rapidly enough at $y=0$ to make only finite contributions to the process under study. For the one loop self-mass-squared the relevant expansions are,

$$
\begin{aligned}
& B(y)=\frac{H^{D-4}}{16 \pi^{\frac{D}{2}}}\left\{-\frac{2 \Gamma\left(\frac{D}{2}-1\right)}{y^{\frac{D}{2}-1}}+\Delta B(y)+O((D-4) \ln (y))\right\} \\
& C(y)=\frac{H^{D-4}}{16 \pi^{\frac{D}{2}}}\left\{-\frac{\Gamma\left(\frac{D}{2}-1\right)}{2 y^{\frac{D}{2}-1}}+\Delta C(y)+O((D-4) \ln (y))\right\}
\end{aligned}
$$


where the $D=4$ residual functions are,

$$
\begin{aligned}
\Delta B(y) & \equiv\left[-\frac{\frac{8}{3}}{(4-y)^{2}}-\frac{\frac{4}{3}}{4-y}\right] \ln \left(\frac{y}{4}\right)-\frac{\frac{2}{3}}{4-y}, \\
\Delta C(y) & \equiv\left[-\frac{\frac{16}{3}}{(4-y)^{3}}-\frac{\frac{4}{3}}{(4-y)^{2}}\right] \ln \left(\frac{y}{4}\right)-\frac{\frac{4}{3}}{(4-y)^{2}}-\frac{\frac{1}{2}}{4-y} .
\end{aligned}
$$

The two combinations for which we require expansions are,

$$
\begin{array}{r}
-(2-y) B-\left(4 y-y^{2}\right) C=\frac{H^{D-4}}{2^{4} \pi^{\frac{D}{2}}}\left\{\frac{4 \Gamma\left(\frac{D}{2}-1\right)}{y^{\frac{D}{2}-1}}+\frac{16 \ln \left(\frac{y}{4}\right)}{(4-y)^{2}}+\frac{\left(\frac{4}{3}+\frac{2}{3} y\right)}{4-y}\right\}, \\
-B+(2-y) C=\frac{H^{D-4}}{2^{4} \pi^{\frac{D}{2}}}\left\{\frac{\Gamma\left(\frac{D}{2}-1\right)}{y^{\frac{D}{2}-1}}+\frac{\frac{32}{3} \ln \left(\frac{y}{4}\right)}{(4-y)^{3}}+\frac{\left(4-\frac{1}{3} y\right)}{(4-y)^{2}}\right\} .
\end{array}
$$

Substituting (171, 172) and (163) in (154) gives,

$$
\begin{aligned}
-i M_{3 \mathrm{AJ}_{1}}^{2}(x ; & \left.x^{\prime}\right)=-i 8 e^{2} H^{2} B a^{D} \delta^{D}\left(x-x^{\prime}\right) \\
+ & \frac{e^{2} H^{2 D-2}}{2^{4} \pi^{D}}\left(a a^{\prime}\right)^{D}\left\{-\frac{8(D-1) \Gamma^{2}\left(\frac{D}{2}\right)}{(D-2) y^{D-2}}-\frac{8}{y(4-y)} \ln \left(\frac{y}{4}\right)\right\} .
\end{aligned}
$$

Although the de Sitter breaking part of the scalar propagator contributes to this expression, it does so in a de Sitter invariant fashion. Explicit breaking of de Sitter invariance appears when we substitute (165) and (163) in (157),

$$
\begin{aligned}
-i M_{3 \mathrm{AJ}_{2+3}}^{2} & \left(x ; x^{\prime}\right)=\frac{e^{2} H^{2 D-2}}{2^{4} \pi^{D}}\left(a a^{\prime}\right)^{D} \\
& \times\left\{\frac{16 \Gamma^{2}\left(\frac{D}{2}\right)}{y^{D-1}}+\frac{4(D-1) \Gamma^{2}\left(\frac{D}{2}\right)}{y^{D-2}}+\frac{2}{y}+a a^{\prime} H^{2} \Delta \eta^{2}\left[\frac{2}{y^{2}}+\frac{1}{y}\right]\right\} .
\end{aligned}
$$

Note that the de Sitter breaking terms are finite so they require no noninvariant counterterms. Because (166) is nonsingular it makes a similarly finite (and similarly non-de Sitter invariant) contribution when substituted with (162) in (159),

$$
\begin{aligned}
-i M_{3 \mathrm{AJ} 4}^{2}(x ; & \left.x^{\prime}\right)=\frac{e^{2} H^{6}}{2^{4} \pi^{4}}\left(a a^{\prime}\right)^{4}\left\{\frac{3}{2} \frac{1}{y}-\frac{3}{4} \ln \left(\frac{\sqrt{e}}{4} H^{2} \Delta x^{2}\right)\right\} \\
+ & \frac{i e^{2} H^{D-2}}{(4 \pi)^{\frac{D}{2}}} \frac{\Gamma(D-1)}{\Gamma\left(\frac{D}{2}\right)}\left\{-\pi \cot \left(\frac{\pi}{2} D\right)+2 \ln (a)\right\} a^{D} \delta^{D}\left(x-x^{\prime}\right) .
\end{aligned}
$$


We can now sum the various terms to obtain the regulated but unrenormalized result,

$$
\begin{aligned}
-i M_{\mathrm{AJ}}^{2}\left(x ; x^{\prime}\right)=+i e^{2} H^{2} 2(D-4) B a^{D} \delta^{D}\left(x-x^{\prime}\right) \\
+\frac{i e^{2} H^{D-2}}{(4 \pi)^{\frac{D}{2}}} \frac{\Gamma(D-1)}{\Gamma\left(\frac{D}{2}\right)}\left\{-\pi \cot \left(\frac{\pi}{2} D\right)+2 \ln (a)\right\} a^{D} \delta^{D}\left(x-x^{\prime}\right) \\
+\frac{e^{2} H^{2 D-2}}{2^{4} \pi^{D}}\left(a a^{\prime}\right)^{D}\left\{\frac{16 \Gamma^{2}\left(\frac{D}{2}\right)}{y^{D-1}}+\frac{4(D-4)(D-1) \Gamma^{2}\left(\frac{D}{2}\right)}{(D-2) y^{D-2}}+\frac{1}{2 y}\right. \\
\left.\quad-\frac{8}{y(4-y)} \ln \left(\frac{y}{4}\right)+a a^{\prime} H^{2} \Delta \eta^{2}\left[\frac{2}{y^{2}}+\frac{1}{y}\right]-\frac{3}{4} \ln \left(\frac{\sqrt{e}}{4} H^{2} \Delta x^{2}\right)\right\} .
\end{aligned}
$$

Only two terms require partial integration,

$$
\begin{aligned}
\frac{e^{2} H^{2 D-2}}{2^{4} \pi^{D}}\left(a a^{\prime}\right)^{D} & \times \frac{16 \Gamma^{2}\left(\frac{D}{2}\right)}{y^{D-1}}=\frac{e^{2}}{4 \pi^{D}} a a^{\prime} \frac{(D-2)^{2} \Gamma^{2}\left(\frac{D}{2}-1\right)}{\Delta x^{2 D-2}} \\
= & \frac{i e^{2} \mu^{D-4}}{4 \pi^{2}} \frac{\Gamma\left(\frac{D}{2}-1\right)}{(D-3)(D-4)} a a^{\prime} \partial^{2} \delta^{D}\left(x-x^{\prime}\right) \\
& \quad-\frac{e^{2}}{2^{5} \pi^{4}} a a^{\prime} \partial^{4}\left(\frac{\ln \left(\mu^{2} \Delta x^{2}\right)}{\Delta x^{2}}\right)+O(D-4) \\
\frac{e^{2} H^{2 D-2}}{2^{4} \pi^{D}}\left(a a^{\prime}\right)^{D} \times & \frac{4(D-4)(D-1) \Gamma^{2}\left(\frac{D}{2}\right)}{(D-2) y^{D-2}} \\
= & \frac{e^{2} H^{2}}{4 \pi^{D}}\left(a a^{\prime}\right)^{2} \frac{(D-4)(D-1) \Gamma^{2}\left(\frac{D}{2}\right)}{(D-2) \Delta x^{2 D-4}} \\
= & \frac{3 i e^{2} H^{2}}{4 \pi^{2}} a^{4} \delta^{4}\left(x-x^{\prime}\right)+O(D-4) .
\end{aligned}
$$

It is also worth noting that the $(D-4)$ times the coincidence limit of $B(y)$ is finite in $D=4$ dimensions,

$$
i e^{2} H^{2} 2(D-4) B a^{D} \delta^{D}\left(x-x^{\prime}\right)=-\frac{i e^{2} H^{2}}{8 \pi^{2}} a^{4} \delta^{4}\left(x-x^{\prime}\right)+O(D-4) .
$$

The simplest choice of renormalization constants seems to be,

$$
\begin{aligned}
\delta Z^{\mathrm{AJ}} & =-\frac{e^{2} \mu^{D-4}}{4 \pi^{\frac{D}{2}}} \frac{\Gamma\left(\frac{D}{2}-1\right)}{(D-3)(D-4)} \\
\delta \xi^{\mathrm{AJ}} & =\frac{1}{4}\left(\frac{D-2}{D-1}\right) \delta Z_{\mathrm{AJ}}-\frac{e^{2} H^{D-4}}{(4 \pi)^{\frac{D}{2}}(D-1) D} \frac{\Gamma(D-1)}{\Gamma\left(\frac{D}{2}\right)}\left\{\pi \cot \left(\frac{\pi}{2} D\right)-5\right\} .
\end{aligned}
$$


Note that the field strength renormalization in Allen-Jacobson gauge is exactly the same (111) that we found for the simple gauge of the previous section. The conformal term in Allen-Jacobson gauge is different from what we found (112) for the simple gauge, but only by finite terms. So the divergence structure seems to be the same.

With these conventions the fully renormalized self-mass-squared is,

$$
\begin{array}{r}
-i M_{\mathrm{A} J_{\mathrm{ren}}}^{2}\left(x ; x^{\prime}\right)=-\frac{i e^{2}}{8 \pi^{2}} a a^{\prime} \ln \left(a a^{\prime}\right) \partial^{2} \delta^{4}\left(x-x^{\prime}\right)+\frac{i e^{2} H^{2}}{4 \pi^{2}} a^{4} \ln (a) \delta^{4}\left(x-x^{\prime}\right) \\
-\frac{e^{2}}{2^{8} \pi^{4}} a a^{\prime} \partial^{6}\left\{\ln ^{2}\left(\mu^{2} \Delta x^{2}\right)-2 \ln \left(\mu^{2} \Delta x^{2}\right)\right\}+\frac{e^{2} H^{6}}{16 \pi^{4}}\left(a a^{\prime}\right)^{4}\left\{\frac{1}{2 y}\right. \\
\left.-\frac{8}{y(4-y)} \ln \left(\frac{y}{4}\right)+a a^{\prime} H^{2} \Delta \eta^{2}\left[\frac{2}{y^{2}}+\frac{1}{y}\right]-\frac{3}{4} \ln \left(\frac{\sqrt{e}}{4} H^{2} \Delta x^{2}\right)\right\}
\end{array}
$$

The first three terms agree with what we got (113) in the simple gauge. The remaining terms show some similarities but they do not agree. Although 1PI functions can differ, off-shell, when computed in different gauges [25], there seems to be a clear disagreement. To see this note that, for large $y\left(x ; x^{\prime}\right)$, the Allen-Jacobson self-mass-squared (184) differs by a factor of $\frac{3}{16} e^{2} H^{4}\left(a a^{\prime}\right)^{4} i \Delta_{A}\left(x ; x^{\prime}\right)$. There is no chance that such a term gives zero when integrated against a wave function, which is how one goes "on-shell" in position space. In fact the integral is singular!

The singularity is not present in (113) so we suspect a problem with the homogeneous terms in the Allen-Jacobson propagator. Specifically, it may not be correct to enforce analyticity at $y=4$. To clarify the situation we will use $i\left[{ }_{\mu} \Delta_{\rho}^{\mathrm{AJ}}\right]\left(x ; x^{\prime}\right)$ to compute the self-mass-squared for a conformally coupled scalar whose propagator is,

$$
i \Delta_{\mathrm{cf}}\left(x ; x^{\prime}\right)=\frac{H^{D-2}}{4 \pi^{\frac{D}{2}}} \frac{\Gamma\left(\frac{D}{2}-1\right)}{y^{\frac{D}{2}-1}} \equiv A_{\mathrm{cf}}(y) .
$$

This field does not experience inflationary particle production, and our result (115) for its self-mass-squared in the simple gauge is a trivial conformal rescaling of the flat space result. That seems to be correct on physical grounds so we can use it to check $i\left[{ }_{\mu} \Delta_{\rho}^{\mathrm{AJ}}\right]\left(x ; x^{\prime}\right)$.

Of course the photon structure is not changed, and most of our previous analysis is still valid. What changes is that the prefactor $k$ of the de Sitter breaking term is zero and, rather than (151), derivatives of the function $A_{\mathrm{cf}}$ 
are all proportional to one another,

$$
A_{\mathrm{cf}}^{\prime}(y)=-\left(\frac{D}{2}-1\right) \frac{A_{\mathrm{cf}}}{y} \quad \text { and } \quad A_{\mathrm{cf}}^{\prime \prime}(y)=\left(\frac{D}{2}-1\right) \frac{D}{2} \frac{A_{\mathrm{cf}}}{y^{2}} .
$$

We require one new combination of the photon functions,

$$
B-(4-y) C=\frac{H^{D-4}}{16 \pi^{\frac{D}{2}}}\left\{\frac{\frac{8}{3}}{(4-y)^{2}} \ln \left(\frac{y}{4}\right)+\frac{\frac{2}{3}}{4-y}+O(D-4)\right\} .
$$

Using these results and (171) one finds,

$$
\begin{gathered}
\left.-i \mathcal{M}_{3 \mathrm{AJ} 1}^{2}\left(x ; x^{\prime}\right) \equiv-4 e^{2} \sqrt{-g} g^{\mu \nu} \sqrt{-g^{\prime}} g^{\prime \rho \sigma} i{ }_{\mu} \Delta_{\rho}^{\mathrm{AJ}}\right]\left(x ; x^{\prime}\right) \partial_{\nu} \partial_{\sigma}^{\prime} i \Delta_{\mathrm{cf}}\left(x ; x^{\prime}\right), \\
=-i 8 e^{2} H^{2} B a^{D} \delta^{D}\left(x-x^{\prime}\right)-4 e^{2} H^{4}\left(a a^{\prime}\right)^{D}\left\{\left[4 D-\left(4 y-y^{2}\right)\right] A_{\mathrm{cf}}^{\prime} B\right. \\
\left.\quad+(2-y)\left(4 y-y^{2}\right) A_{\mathrm{cf}}^{\prime} C+(2-y)\left(4 y-y^{2}\right) A_{\mathrm{cf}}^{\prime \prime} B+\left(4 y-y^{2}\right)^{2} A_{\mathrm{cf}}^{\prime \prime} C\right\}, \\
=-i 8 e^{2} H^{2} B a^{D} \delta^{D}\left(x-x^{\prime}\right)+2(D-2) e^{2} H^{4}\left(a a^{\prime}\right)^{D} A_{\mathrm{cf}} \\
\quad \times\left\{2(D-1)[B-(4-y) C]+\left(\frac{D}{2}-1\right)\left[(2-y) B+\left(4 y-y^{2}\right) C\right]\right\}, \\
=-i 8 e^{2} H^{2} B a^{D} \delta^{D}\left(x-x^{\prime}\right) \\
\quad+\frac{e^{2} H^{2 D-2}}{16 \pi^{D}}\left(a a^{\prime}\right)^{D}\left\{-\frac{4 \Gamma^{2}\left(\frac{D}{2}\right)}{y^{D-2}}+\frac{2}{3} \frac{1}{y}+O(D-4)\right\} .
\end{gathered}
$$

The divergence on $x^{\nu}$ gives,

$$
\begin{gathered}
-i \mathcal{M}_{3 \mathrm{AJ}_{2}}^{2}\left(x ; x^{\prime}\right) \equiv-2 e^{2} \partial_{\nu}\left(\sqrt{-g} g^{\mu \nu} i\left[{ }_{\mu} \Delta_{\rho}^{\mathrm{AJ}}\right]\left(x ; x^{\prime}\right)\right) \sqrt{-g^{\prime} g^{\prime \rho \sigma}} \partial_{\sigma}^{\prime} i \Delta_{\mathrm{cf}}\left(x ; x^{\prime}\right) \\
=(D-2) e^{2} H^{4}\left(a a^{\prime}\right)^{D}(4-y) A_{\mathrm{cf}}\left\{-\left(4 y-y^{2}\right) F^{\prime}-(D-1)(2-y) F\right\} .
\end{gathered}
$$

This is symmetric under interchange of $x^{\mu}$ and $x^{\prime \mu}$ so one gets the same from the divergence on $x^{\prime \sigma}$, and the two divergence terms sum to,

$$
\begin{aligned}
-i \mathcal{M}_{3 \mathrm{AJ}_{2}+3}^{2}\left(x ; x^{\prime}\right)=2(D-2) e^{2} H^{4}\left(a a^{\prime}\right)^{D}(4-y) A_{\mathrm{cf}} & \\
& \times\left\{-\left(4 y-y^{2}\right) F^{\prime}-(D-1)(2-y) F\right\} . \\
= & \frac{e^{2} H^{2 D-2}}{16 \pi^{D}}\left\{\frac{16 \Gamma^{2}\left(\frac{D}{2}\right)}{y^{D-1}}+\frac{2(D-2) \Gamma^{2}\left(\frac{D}{2}\right)}{y^{D-2}}-\frac{2}{y}+O(D-4)\right\} .
\end{aligned}
$$


Because the coincidence limit of $A_{\mathrm{cf}}(y)$ vanishes in dimensional regularization we obtain no local contribution from the last term in (146),

$$
\begin{aligned}
& -i \mathcal{M}_{3 \mathrm{AJ} 4}^{2}\left(x ; x^{\prime}\right) \equiv-e^{2} \partial_{\nu} \partial_{\sigma}^{\prime}\left(\sqrt{-g} g^{\mu \nu} \sqrt{-g^{\prime}} g^{\prime \rho \sigma} i\left[{ }_{\mu} \Delta_{\rho}^{\mathrm{AJ}}\right]\left(x ; x^{\prime}\right)\right) i \Delta_{\mathrm{cf}}\left(x ; x^{\prime}\right) \\
& =(D-1) e^{2} H^{4}\left(a a^{\prime}\right)^{D} A_{\mathrm{cf}}\left\{(2-y)\left(4 y-y^{2}\right) F^{\prime}+(D-1)(2-y)^{2} F+4 F\right\}, \\
& =\frac{e^{2} H^{6}}{16 \pi^{4}}\left\{\frac{3}{2} \frac{1}{y}+O(D-4)\right\} .
\end{aligned}
$$

Because it does not involve the scalar, the 4-point contribution is unchanged,

$$
\begin{aligned}
-i \mathcal{M}_{4 \mathrm{AJ}}^{2}\left(x ; x^{\prime}\right) & \equiv-i e^{2} \sqrt{-g} g^{\mu \nu} i\left[{ }_{\mu} \Delta_{\nu}^{\mathrm{AJ}}\right]\left(x ; x^{\prime}\right) \delta^{D}\left(x-x^{\prime}\right), \\
& =i 2 D e^{2} H^{2} B a^{D} \delta^{D}\left(x-x^{\prime}\right) .
\end{aligned}
$$

It remains to sum the various contributions and isolate the divergences,

$$
\begin{aligned}
& -i \mathcal{M}_{\mathrm{AJ}}^{2}\left(x ; x^{\prime}\right)=i 2(D-4) e^{2} H^{2} B a^{D} \delta^{D}\left(x-x^{\prime}\right) \\
& +\frac{e^{2} H^{2 D-2}}{16 \pi^{D}}\left(a a^{\prime}\right)^{D}\left\{\frac{16 \Gamma^{2}\left(\frac{D}{2}\right)}{y^{D-1}}+\frac{2(D-4) \Gamma^{2}\left(\frac{D}{2}\right)}{y^{D-2}}+\frac{1}{6 y}+O(D-4)\right\} \\
& =\frac{i e^{2} \mu^{D-4}}{4 \pi^{\frac{D}{2}}} \frac{\Gamma\left(\frac{D}{2}-1\right)}{(D-3)(D-4)} a a^{\prime} \partial^{2} \delta^{D}\left(x-x^{\prime}\right)+\frac{i e^{2} H^{2}}{8 \pi^{2}} a^{4} \delta^{4}\left(x-x^{\prime}\right) \\
& -\frac{e^{2}}{2^{8} \pi^{4}} a a^{\prime} \partial^{6}\left\{\ln ^{2}\left(\mu^{2} \Delta x^{2}\right)-2 \ln \left(\mu^{2} \Delta x^{2}\right)\right\}+\frac{e^{2} H^{4}}{96 \pi^{4}} \frac{\left(a a^{\prime}\right)^{3}}{\Delta x^{2}}+O(D-4) .
\end{aligned}
$$

The best choice of counterterms would seem to be,

$$
\begin{aligned}
\left.\delta Z_{2}^{\mathrm{AJ}}\right|_{\mathrm{conf}} & =-\frac{e^{2} \mu^{D-4}}{4 \pi^{\frac{D}{2}}} \frac{\Gamma\left(\frac{D}{2}-1\right)}{(D-3)(D-4)} \\
\left.\delta \xi^{\mathrm{AJ}}\right|_{\text {conf }} & =\frac{e^{2} \mu^{D-4}}{4 \pi^{\frac{D}{2}}(D-1) D}\left\{-\frac{\Gamma\left(\frac{D}{2}+1\right)}{(D-3)(D-4)}+\frac{1}{2}\right\}
\end{aligned}
$$

This choice gives the following renormalized self-mass-squared,

$$
\begin{aligned}
-i \mathcal{M}_{\mathrm{AJ} \text { ren }}^{2}\left(x ; x^{\prime}\right) & =-\frac{i e^{2}}{8 \pi^{2}} a a^{\prime} \ln \left(a a^{\prime}\right) \partial^{2} \delta^{4}\left(x-x^{\prime}\right) \\
& -\frac{e^{2}}{2^{8} \pi^{4}} a a^{\prime} \partial^{6}\left\{\ln ^{2}\left(\mu^{2} \Delta x^{2}\right)-2 \ln \left(\mu^{2} \Delta x^{2}\right)\right\}+\frac{e^{2} H^{4}}{96 \pi^{4}} \frac{\left(a a^{\prime}\right)^{3}}{\Delta x^{2}} .
\end{aligned}
$$


The first two terms agree with what we found (115) in the simple gauge, but the final term is again proportional to $\left(a a^{\prime}\right)$ times the (conformal) scalar propagator.

How did this happen? The conformal scalar propagator is of course correct, as is the expression (146) for the 3-point contribution. It might seem that a bewildering sequence of expansions have taken place but the astute reader will note that, except for local terms, the entire result is proportional to the factor,

$$
2(D-2) e^{2} H^{4}\left(a a^{\prime}\right)^{D} A_{\mathrm{cf}}(y)=\frac{e^{2} H^{D+2}}{\pi^{\frac{D}{2}}}\left(a a^{\prime}\right)^{D} \frac{\Gamma\left(\frac{D}{2}\right)}{y^{\frac{D}{2}-1}},
$$

times the sum of just three combinations of the functions $B(y), C(y)$ and $F(y)$. The three key combinations can be read off from expressions (190), (193) and (195),

$$
\begin{aligned}
& 2(D-1)[B-(4-y) C]+\left(\frac{D}{2}-1\right)\left[(2-y) B+\left(4 y-y^{2}\right) C\right] \\
& =\frac{H^{D-4}}{16 \pi^{\frac{D}{2}}}\left\{-\frac{4 \Gamma\left(\frac{D}{2}\right)}{y^{\frac{D}{2}-}}+\frac{2}{3}+O(D-4)\right\} \\
& -(4-y)\left[\left(4 y-y^{2}\right) F^{\prime}+(D-1)(2-y) F\right] \\
& =\frac{H^{D-4}}{16 \pi^{\frac{D}{2}}}\left\{\frac{16 \Gamma\left(\frac{D}{2}\right)}{y^{\frac{D}{2}}}+\frac{2(D-2) \Gamma\left(\frac{D}{2}\right)}{y^{\frac{D}{2}-1}}-2+O(D-4)\right\} \\
& \frac{1}{2}\left(\frac{D-1}{D-2}\right)\left[(2-y)\left(4 y-y^{2}\right) F^{\prime}+(D-1)(2-y)^{2} F+4 F\right] \\
& =\frac{H^{D-4}}{16 \pi^{\frac{D}{2}}}\left\{\frac{3}{2}+O(D-4)\right\}
\end{aligned}
$$

The problem derived from the presence of the constant terms in each of these three expansions. Note that it would not even be acceptable to have three nonzero constants sum to zero because (206) and (207) contribute as well to the minimally coupled self-mass-squared. Indeed, in that case the worst problem derives from the nonzero constant in just (207). Whatever we do must therefore expunge these constant terms.

What freedom do we have to alter $B(y), C(y)$ and $F(y)$ ? First recall that $C(y)$ is not independent, but instead obeys $C(y)=B^{\prime}(y)-F(y)$. The functions $B(y)$ and $F(y)$ must of course satisfy the equations (135] 136) which 
define the photon propagator. However, we suspect a homogeneous solution is missing. Because two of the three crucial combinations involve only $F(y)$ we begin with it. The two independent solutions to the $F$ equation equation (135) and their $D=4$ limits are,

$$
\begin{aligned}
& F_{1}(y) \equiv \frac{1}{16}{ }_{2} F_{1}\left(D-1,2 ; \frac{D}{2}+1 ; 1-\frac{y}{4}\right) \longrightarrow \frac{1}{y^{2}} \\
& F_{2}(y) \equiv \frac{1}{16}{ }_{2} F_{1}\left(D-1,2 ; \frac{D}{2}+1 ; \frac{y}{4}\right) \longrightarrow \frac{1}{(4-y)^{2}} .
\end{aligned}
$$

In $D=4$ these induce the following dependence in $B(y)$ through its equation (136),

$$
\begin{aligned}
& \delta B_{1}(y)=-\frac{1}{y}-\left[\frac{\frac{4}{3}}{(4-y)^{2}}+\frac{\frac{2}{3}}{4-y}\right] \ln \left(\frac{y}{4}\right)-\frac{\frac{1}{3}}{4-y}, \\
& \delta B_{2}(y)=\frac{1}{4-y}+\left[\frac{\frac{4}{3}}{y^{2}}+\frac{\frac{2}{3}}{y}\right] \ln \left(1-\frac{y}{4}\right)+\frac{\frac{1}{3}}{y} .
\end{aligned}
$$

The solution of Allen and Jacobson is entirely based upon $F_{1}(y)$ and $\delta B_{1}(y)$. The other solution, $F_{2}(y)$ and $\delta B_{2}(y)$, was rejected on the grounds that its singularity at the antipodal point (the pole at $y=4$ ) would correspond to a point source there. Although this seemed correct to us as well, the problem we have encountered with the self-mass-squared makes us suspect that, on the full de Sitter manifold, the photon propagator should have an anti-source at the antipodal point $\bar{x}^{\mu}$,

$$
\sqrt{-g}\left[\left(D^{2}\right)_{\rho}^{\mu}-R_{\rho}^{\mu}\right] i\left[{ }^{\rho} \Delta_{\nu}\right]\left(x ; x^{\prime}\right)=\delta_{\nu}^{\mu} i\left[\delta^{D}\left(x-x^{\prime}\right)-\delta^{D}\left(\bar{x}-x^{\prime}\right)\right] .
$$

We offer two arguments in support of this suggestion. First, the linearization instability of the gauge invariant theory precludes solutions with nonzero charge, and the only de Sitter invariant position at which to locate the compensating anti-charge is the antipodal point. Second, including an anti-source makes the problem go away. The contributions of $-F_{2}(y) / 8 \pi^{2}$, and of the terms it induces in $B(y)$ and $C(y)$, to expressions (205) 207) are,

$$
\begin{array}{r}
-\frac{1}{8 \pi^{2}}\left\{2(D-1)\left[\delta B_{2}-(4-y) \delta C_{2}\right]+\left(\frac{D}{2}-1\right)\left[(2-y) \delta B_{2}+\left(4 y-y^{2}\right) \delta C_{2}\right]\right\} \\
=\frac{H^{D-4}}{16 \pi^{\frac{D}{2}}}\left\{-\frac{8}{4-y}-\frac{128}{y^{3}} \ln \left(1-\frac{y}{4}\right)-\frac{32}{y^{2}}-\frac{4}{y}-\frac{2}{3}+O(D-4)\right\},(2
\end{array}
$$




$$
\begin{gathered}
-\frac{1}{8 \pi^{2}} \times-(4-y)\left[\left(4 y-y^{2}\right) F_{2}^{\prime}+(D-1)(2-y) F_{2}\right] \\
=\frac{H^{D-4}}{16 \pi^{\frac{D}{2}}}\left\{\frac{4}{4-y}+2+O(D-4)\right\}, \\
-\frac{1}{8 \pi^{2}} \times \frac{1}{2}\left(\frac{D-1}{D-2}\right)\left[(2-y)\left(4 y-y^{2}\right) F_{2}^{\prime}+(D-1)(2-y)^{2} F_{2}+4 F_{2}\right] \\
=\frac{H^{D-4}}{16 \pi^{\frac{D}{2}}}\left\{-\frac{3}{2}+O(D-4)\right\} .
\end{gathered}
$$

A final point is that there is nothing to be gained from the homogeneous solutions to the $B$ equation (136),

$$
\begin{aligned}
B_{1}(y) & \equiv \frac{1}{16}{ }_{2} F_{1}\left(D, 1 ; \frac{D}{2}+1 ; 1-\frac{y}{4}\right) \longrightarrow \frac{1}{y^{2}}+\frac{\frac{1}{2}}{y} \\
B_{2}(y) & \equiv \frac{1}{16}{ }_{2} F_{1}\left(D, 1 ; \frac{D}{2}+1 ; \frac{y}{4}\right) \longrightarrow \frac{1}{(4-y)^{2}}+\frac{\frac{1}{2}}{4-y} .
\end{aligned}
$$

Of course we must reject $B_{1}(y)$ on account of its singularity at $y=0$. Because we do not care overmuch what happens at the antipodal point we might allow $B_{2}(y)$. However, this does not affect relations (206,207) because they involve only $F(y)$. It turns out that $B_{2}(y)$ also has no effect upon (205), so there is nothing to be gained by adding it.

\section{Discussion}

We have used two different gauges to compute the renormalized self-masssquared for a massless, minimally coupled scalar and for a massless, conformally coupled scalar. In the simplest gauge [16] our result for the minimally coupled scalar is,

$$
\begin{aligned}
& M_{\text {ren }}^{2}\left(x ; x^{\prime}\right)=\frac{e^{2}}{8 \pi^{2}} a a^{\prime} \ln \left(a a^{\prime}\right) \partial^{2} \delta^{4}\left(x-x^{\prime}\right)-\frac{e^{2} H^{2}}{4 \pi^{2}} a^{4} \ln (a) \delta^{4}\left(x-x^{\prime}\right) \\
& -\frac{i e^{2}}{2^{8} \pi^{4}} a a^{\prime} \partial^{6}\left\{\ln ^{2}\left(\mu^{2} \Delta x^{2}\right)-2 \ln \left(\mu^{2} \Delta x^{2}\right)\right\}-\frac{i e^{2} H^{4}}{2^{6} \pi^{4}}\left(a a^{\prime}\right)^{3}\left\{\partial _ { 0 } ^ { 2 } \left[\ln ^{2}\left(2^{-2} H^{2} \Delta x^{2}\right)\right.\right. \\
& \left.\left.\quad-\ln \left(2^{-2} H^{2} \Delta x^{2}\right)\right]+\frac{\partial^{2}}{2}\left[3 \ln ^{2}\left(2^{-2} H^{2} \Delta x^{2}\right)-5 \ln \left(2^{-2} H^{2} \Delta x^{2}\right)\right]\right\} .
\end{aligned}
$$


Although this gauge breaks de Sitter invariance, it did not require any noninvariant counterterms. Nor did it prevent the attainment of a fully de Sitter invariant result for the conformally coupled scalar,

$$
\begin{aligned}
\mathcal{M}_{\text {ren }}^{2}\left(x ; x^{\prime}\right)=\frac{e^{2}}{8 \pi^{2}} a a^{\prime} \ln \left(a a^{\prime}\right) \partial^{2} \delta^{4}\left(x-x^{\prime}\right) & \\
& -\frac{i e^{2}}{2^{8} \pi^{4}} a a^{\prime} \partial^{6}\left\{\ln ^{2}\left(\mu^{2} \Delta x^{2}\right)-2 \ln \left(\mu^{2} \Delta x^{2}\right)\right\} .
\end{aligned}
$$

Working in a de Sitter invariant gauge [18] was much more complicated. Although all contractions and derivatives of de Sitter invariant objects produce simple combinations of the propagator functions $B(y)$ and $C(y)$ and their derivatives, the propagator functions (137) 138) are themselves so complicated as to preclude a simple analysis. It is particularly pointless to employ a de Sitter invariant gauge when the matter field in question necessarily breaks de Sitter invariance as does the minimally coupled scalar [17].

There also seems to be a physical problem with the de Sitter invariant propagator. This is evident from our renormalized results for the minimally coupled and conformally coupled scalars,

$$
\begin{aligned}
& M_{\mathrm{AJ} \text { ren }}^{2}\left(x ; x^{\prime}\right)=\frac{e^{2}}{8 \pi^{2}} a a^{\prime} \ln \left(a a^{\prime}\right) \partial^{2} \delta^{4}\left(x-x^{\prime}\right)-\frac{e^{2} H^{2}}{4 \pi^{2}} a^{4} \ln (a) \delta^{4}\left(x-x^{\prime}\right) \\
&-\frac{i e^{2}}{2^{8} \pi^{4}} a a^{\prime} \partial^{6}\left\{\ln ^{2}\left(\mu^{2} \Delta x^{2}\right)-2 \ln \left(\mu^{2} \Delta x^{2}\right)\right\}+\frac{i e^{2} H^{6}}{16 \pi^{4}}\left(a a^{\prime}\right)^{4}\left\{\frac{1}{2 y}\right. \\
&\left.-\frac{8}{y(4-y)} \ln \left(\frac{y}{4}\right)+a a^{\prime} H^{2} \Delta \eta^{2}\left[\frac{2}{y^{2}}+\frac{1}{y}\right]-\frac{3}{4} \ln \left(\frac{\sqrt{e}}{4} H^{2} \Delta x^{2}\right)\right\} \\
& \mathcal{M}_{\mathrm{AJ} \text { ren }}^{2}\left(x ; x^{\prime}\right)= \frac{e^{2}}{8 \pi^{2}} a a^{\prime} \ln \left(a a^{\prime}\right) \partial^{2} \delta^{4}\left(x-x^{\prime}\right) \\
&-\frac{i e^{2}}{2^{8} \pi^{4}} a a^{\prime} \partial^{6}\left\{\ln ^{2}\left(\mu^{2} \Delta x^{2}\right)-2 \ln \left(\mu^{2} \Delta x^{2}\right)\right\}+\frac{i e^{2} H^{4}}{96 \pi^{4}} \frac{\left(a a^{\prime}\right)^{3}}{\Delta x^{2}} .
\end{aligned}
$$

In each case the leading infrared contribution goes like $\left(a a^{\prime}\right)^{4}$ times the relevant scalar propagator. Although off-shell quantities such as the self-masssquared can depend upon the choice of gauge [25], some of the extra terms we get for the de Sitter invariant gauge are actually singular on shell.

We suspect the problem is that the de Sitter invariant propagator should not be required to be analytic at the antipodal point. We demonstrated that the on-shell singularities disappear when the propagator is given an antisource antipodal to the source. There may be a mathematical justification 
for this in the fact that the gauge invariant theory possesses a linearization instability which precludes solutions with nonzero net charge.

Of course this study was not undertaken to compare different gauges! Our motivation was instead to learn how the scalar responds to the dielectric medium of super-horizon scalars produced by inflation. It has previously been shown that this medium causes super-horizon photons to behave, in some ways, as if they have positive mass [2, 9, 10, 11. Key questions we seek to answer are:

- Does part of the scalar get "eaten" to make up the longitudinal polarization of the massive photon as in the Higgs mechanism?

- Does the one loop self-mass-squared enhance or retard further particle production?

Both questions can be answered by using the self-mass-squared (218) to solve the linearized effective field equation (11) at one loop order. This will be undertaken in a subsequent paper.

A final application of this computation is to serve as "data" in obtaining a stochastic formulation of SQED to sum the leading infrared logarithms [26. 27. Aside from its intrinsic interest, SQED provides a nearly perfect arena for the difficult task of generalizing Starobinskilı's techniques [28, 29] from simple scalar models to quantum gravity. It possesses derivative interactions and gauge constraints, it shows infrared logarithms and it is relatively tractable. This exercise has demonstrated the validity and viability of the Feynman rules in the noninvariant gauge. The stage has been set for the computations which are crucial for checking any stochastic formulation of SQED, the two loop vacuum expectation values,

$$
\left\langle\Omega\left|\varphi^{*}(x) \varphi(x)\right| \Omega\right\rangle \quad, \quad\left\langle\Omega\left|F_{\rho \sigma}(x) F_{\mu \nu}(x)\right| \Omega\right\rangle \quad \text { and } \quad\left\langle\Omega\left|T_{\mu \nu}(x)\right| \Omega\right\rangle .
$$

\section{Acknowledgments}

This work was partially supported by NSF grant PHY-0244714 and by the Institute for Fundamental Theory at the University of Florida.

\section{References}

[1] L. Parker, Phys. Rev. 183 (1969) 1057. 
[2] T. Prokopec and R. P. Woodard, Am. J. Phys. 72 (2004) 60, astro-ph/0303358.

[3] R. P. Woodard, "Fermion Self-Energy during Inflation," Selected Problems of Modern Physics," ed. B. M. Barbashov, G. V. Efimov, A. V. Efremov, S. M. Eliseev, V. V. Nestorenko and M. K. Volkov (Dubna, JINR, 2003) pp. 355-366, astro-ph/0307269.

[4] R. P. Woodard, "Quantum Effects during Inflation," in Norman 2003, Quantum field theory under the influence of external conditions," ed. K. A. Milton (Rinton Press, Princeton, 2004) pp. 325-330, astro-ph/0310757.

[5] V. F. Mukhanov and G. V. Chibisov, JETP Lett. 33 (1981) 532.

[6] A. A. Starobinskiı̌, JETP Lett. 30 (1979) 682.

[7] V. Mukhanov, H. Feldman and R. Brandenberger, Phys. Rep. 215 (1992) 203.

[8] A. R. Liddle and D. H. Lyth, Phys. Rep. 231 (1993) 1, astro-ph/9303019 .

[9] T. Prokopec, O. Törnkvist and R. P. Woodard, Phys. Rev. Lett. 89 (2002) 101301, astro-ph/0205331.

[10] T. Prokopec, O. Törnkvist and R. P. Woodard, Ann. Phys. 303 (2003) 251, gr-qc/0205130.

[11] T. Prokopec and R. P. Woodard, Ann. Phys. 312 (2204) 1, astro-ph/0310757.

[12] T. Prokopec and R. P. Woodard, JHEP 0310 (2003) 059, astro-ph/0309593.

[13] A. C. Davis, K. Dimopoulos, T. Prokopec and O. Törnkvist, Phys. Lett. B501 (2001) 165, astro-ph/0007214.

[14] K. Dimopoulos, T. Prokopec, O. Törnkvist and A. C. Davis, Phys. Rev. D65 (2002) 063505, astro-ph/0108093. 
[15] L. D. Duffy and R. P. Woodard, Phys. Rev. D72 (2005) 024023, hep-ph/0505156.

[16] R. P. Woodard, "de Sitter Breaking in Field Theory," gr-qc/0408002.

[17] B. Allen and A. Follaci, Phys. Rev. D35 (1987) 3771.

[18] B. Allen and T. Jacobson, Commun. Math, Phys. 103 (1986) 669.

[19] N. D. Birrell and P. C. W. Davies, Quantum Fields in Curved Space (Cambridge University Press, Cambridge, 1982).

[20] B. Allen, Phys. Rev. D32 (1985) 3136.

[21] V. K. Onemli and R. P. Woodard, Class. Quant. Grav. 19 (2002), 4607, gr-qc/0204065.

[22] V. K. Onemli and R. P. Woodard, Phys Rev. D70 (2004) 107301, gr-qc/0406098.

[23] P. Candelas and D. J. Raine, Phys. Rev. D12 (1975) 965.

[24] J. S. Dowker and R. Critchley, Phys. Rev. D13 (1976) 3224.

[25] R. Jackiw, Phys. Rev. D9 (1974) 1686.

[26] R. P. Woodard, Nucl. Phys. Proc. Suppl. 148 (2005), 108, astro-ph/0502556.

[27] N. C. Tsamis and R. P. Woodard, "Stochastic Quantum Gravitational Inflation," Nucl. Phys. B in press, gr-qc/0505115.

[28] A. A. Starobinskiu, "Stochastic de Sitter (inflationary) stage in the early universe," in Field Theory, Quantum Gravity and Strings, ed. H. J. de Vega and N. Sanchez (Springer-Verlag, Berlin, 1986) pp. 107-126.

[29] A. A. Starobinskiŭ and J. Yokoyama, Phys. Rev. D50 (1994) 6357, astro-ph/9407016. 